\title{
Existence of weak solutions for unsteady motions of generalized Newtonian fluids
}

\author{
LARS DIENING, MICHAEL RŮŽIČKA AND JÖRG WOLF
}

\begin{abstract}
We prove the existence of weak solutions $\mathbf{u}: Q_{T} \rightarrow \mathbb{R}^{n}$ of the equations of unsteady motion of an incompressible fluid with shear-dependent viscosity in a cylinder $Q_{T}=\Omega \times(0, T)$, where $\Omega \subset \mathbb{R}^{n}$ denotes a bounded domain. Under the assumption that the extra stress tensor $\mathbf{S}$ possesses a $q$-structure with $q>\frac{2 n}{n+2}$, we are able to construct a weak solution $\mathbf{u} \in L^{q}\left(0, T ; W_{0}^{1, q}(\Omega)\right) \cap$ $C_{w}\left([0, T] ; L^{2}(\Omega)\right)$ with $\operatorname{div} \mathbf{u}=0$. Our approach is based on the Lipschitz truncation method, which is new in this context.
\end{abstract}

Mathematics Subject Classification (2000): 76D03 (primary);35D05,46,34A34 (secondary).

\section{Introduction. Statement of the main result}

Let $\Omega \subset \mathbb{R}^{n}, n \geq 2$, be a bounded domain. For $0<T<\infty$ we set $Q_{T}:=$ $\Omega \times(0, T)$. The isothermal motion of a homogeneous, incompressible fluid through $\Omega$ is governed by the balance equations for linear momentum and mass, which read ${ }^{1}$

$$
\begin{aligned}
& \rho \partial_{t} \mathbf{u}+\rho \operatorname{div}(\mathbf{u} \otimes \mathbf{u})-\operatorname{div} \mathbf{S}+\nabla p=\rho \mathbf{f} \text { in } \quad Q_{T}, \\
& \operatorname{div} \mathbf{u}=0 \quad \text { in } \quad Q_{T},
\end{aligned}
$$

where $\mathbf{u}=\left(u^{1}, \ldots, u^{n}\right)$ is the velocity, $p$ the pressure, $\mathbf{S}=\left(S_{i j}\right)_{i, j=1}^{n}$ the extra stress tensor, $\mathbf{f}=\left(f^{1}, \ldots, f^{n}\right)$ the external body force and $\rho$ the constant density. In the following we divide the equation (1.1) by the constant density $\rho$ and relabel $\mathbf{S} / \rho$ and $p / \rho$ again as $\mathbf{S}$ and $p$, respectively. Moreover, for mathematical simplicity we assume that $\mathbf{f}=\operatorname{div} \mathbf{F}$, where $\mathbf{F}=\left(F_{i j}\right)_{i, j=1}^{n}$ is a given tensor. The above system (1.1), (1.2) has to be completed by boundary and initial conditions and by constitutive assumptions for the extra stress tensor. Concerning the former we assume at

${ }^{1}$ Here, $\operatorname{div} \mathbf{v}:=\sum_{i} \partial_{x_{i}} v^{i}$, where $\partial_{x_{i}}=\frac{\partial}{\partial x_{i}}(i=1, \ldots, n)$.

Received June 3, 2008; accepted in revised form March 10, 2009. 
the initial time $t=0$

$$
\mathbf{u}(0)=\mathbf{u}_{0} \quad \text { in } \Omega,
$$

where $\mathbf{u}_{0}$ denotes the initial velocity, which is a given vector field with div $\mathbf{u}_{0}=0$. On the boundary $\partial \Omega$ we assume the following condition of adherence

$$
\mathbf{u}=\mathbf{0} \quad \text { on } \partial \Omega \times(0, T) .
$$

If one assumes a linear dependence of the extra stress tensor $\mathbf{S}$ on the symmetric part of the velocity gradient $\mathbf{D}=\mathbf{D}(\mathbf{u}):=\frac{1}{2}\left(\nabla \mathbf{u}+\nabla \mathbf{u}^{\top}\right)$, then system (1.1)(1.2) is just the classical Navier-Stokes equations. However, there exists many homogeneous, incompressible fluids that cannot be adequately described by such a simple constitutive relation. Such fluids are usually called non-Newtonian fluids. There are many ways in which a non-Newtonian behaviour can manifest itself and we refer the reader to [4] and [26] for a general continuum mechanical background and to $[3,6,23,44,50]$, and [35] for a detailed discussion of non-Newtonian fluids. There is a large class of non-Newtonian fluids for which the dominant departure from a Newtonian behaviour is that in a simple shear flow the viscosity and the shear rate are not proportional. Such fluids are called fluids with shear-dependent viscosity or generalized Newtonian fluids and are often modeled by the constitutive law

$$
\mathbf{S}=\mu\left(\mathbf{D}_{I I}\right) \mathbf{D}
$$

where $\mathbf{D}_{I I}=\mathbf{D}: \mathbf{D}$ is the second invariant ${ }^{2}$ of $\mathbf{D}$ and $\mu$ is the generalized viscosity function. The model (1.5) includes all power-law and Carreau-type models, which are quite popular among rheologists. Such models are used in many areas of engineering sciences such as chemical engineering, colloidal mechanics, glaciology, geology, and blood rheology ( $c f$. [36] for a discussion of such models and further references). Typical examples for the constitutive relation (1.5) are

$$
\begin{aligned}
& \mathbf{S}=\mu_{0}(\delta+|\mathbf{D}|)^{q-2} \mathbf{D}, \\
& \mathbf{S}=\mu_{0}\left(\delta^{2}+|\mathbf{D}|^{2}\right)^{\frac{q-2}{2}} \mathbf{D},
\end{aligned}
$$

with $1<q<\infty, \delta \geq 0$ and $\mu_{0}>0$.

Motivated by the above discussion we impose the following conditions on $\mathbf{S}$.

We say that the extra stress tensor $\mathbf{S}$ possesses a $q$-structure if there exist $q \in(1, \infty)$ and $\delta \geq 0$, such that

(I) $\mathbf{S}: Q_{T} \times \mathbb{M}_{\text {sym }}^{n} \rightarrow \mathbb{M}_{\text {sym }}^{n}$ is a Carathéodory function ${ }^{3}$;

${ }^{2}$ For two matrices $\mathbf{A}, \mathbf{B} \in \mathbb{R}^{n^{2}}$ by $\mathbf{A}: \mathbf{B}$ we denote the sum $\sum_{i j} A_{i j} B_{i j}$.

$3 \mathbb{M}_{\text {sym }}^{n}$ is the vector space of all symmetric $n \times n$ matrices $\xi=\left(\xi_{i j}\right)_{i, j=1}^{n}$. We equip $\mathbb{M}_{\text {sym }}^{n}$ with the scalar product $\xi: \eta$ and the norm $|\xi|:=(\xi: \xi)^{1 / 2}$. By $\mathbf{a} \cdot \mathbf{b}$ we denote the usual scalar product in $\mathbb{R}^{n}$ and by $|\mathbf{a}|$ we denote the Euclidean norm. 
Growth condition:

(II) $|\mathbf{S}(x, t, \boldsymbol{\xi})| \leq c_{0}(\delta+|\boldsymbol{\xi}|)^{q-2}|\boldsymbol{\xi}|+\kappa_{1}(x, t) \quad$ for all $\quad \boldsymbol{\xi} \in \mathbb{M}_{\text {sym }}^{n}$, almost all $(x, t) \in Q_{T}\left(c_{0}>0, \kappa_{1} \in L^{q^{\prime}}\left(Q_{T}\right), \kappa_{1} \geq 0\right) ;$

Coercivity:

(III) $\mathbf{S}(x, t, \xi): \xi \geq v_{0}(\delta+|\xi|)^{q-2}|\xi|^{2}-\kappa_{2}(x, t) \quad$ for all $\boldsymbol{\xi} \in \mathbb{M}_{\text {sym }}^{n}$, almost all $(x, t) \in Q_{T}\left(v_{0}>0, \kappa_{2} \in L^{1}\left(Q_{T}\right), \kappa_{2} \geq 0\right) ;$

Strict monotonicity:

(IV)

$$
\left\{\begin{array}{l}
(\mathbf{S}(x, t, \boldsymbol{\xi})-\mathbf{S}(x, t, \boldsymbol{\eta})):(\boldsymbol{\xi}-\boldsymbol{\eta})>0 \\
\forall \xi, \boldsymbol{\eta} \in \mathbb{M}_{\text {sym }}^{n}(\xi \neq \boldsymbol{\eta}), \text { almost all }(x, t) \in Q_{T}
\end{array}\right.
$$

Before we introduce the notion of a weak solution of the system (1.1)-(1.4) let us provide some notation and function spaces which will be used throughout the paper. As usual let $C_{0}^{\infty}(\Omega)$ denote the space of all smooth functions having compact support in $\Omega$. By $W^{k, q}(\Omega), k \in \mathbb{N}, 1 \leq q \leq \infty$, we denote the usual Sobolev spaces. We define $W_{0}^{k, q}(\Omega)$ as the closure of $C_{0}^{\infty}(\Omega)$ in $W^{k, q}(\Omega)$. We will not distinguish between scalar-valued, vector-valued or tension-valued versions of these function spaces. The Lebesgue measure in $\mathbb{R}^{n+1}$ will be denoted by $\mathcal{L}_{n+1}(\cdot)$. For $A \subset \mathbb{R}^{n+1}$ with $0<\mathcal{L}_{n+1}(A)<\infty$ and $g \in L_{\text {loc }}^{1}\left(\mathbb{R}^{n+1}\right)$ we denote by $g_{A}$ the mean value of $g$ over the set $A$, i.e.

$$
v_{A}:=f_{A} v \mathrm{~d} X=\frac{1}{\mathcal{L}_{n+1}(A)} \int_{A} v \mathrm{~d} X .
$$

Let $\left(X,\|\cdot\|_{X}\right)$ be a normed space. By $L^{q}(0, T ; X)$ we denote the space of all Bochner measurable functions $\varphi:(0, T) \rightarrow X$, such that

$$
\begin{aligned}
& \|\varphi\|_{L^{q}(0, T ; X)}:=\left(\int_{0}^{T}\|\varphi(t)\|_{X}^{q} \mathrm{~d} t\right)^{\frac{1}{q}}<+\infty \quad \text { if } \quad 1 \leq q<+\infty, \\
& \|\varphi\|_{L^{\infty}(0, T ; X)}:=\operatorname{ess} \sup \|\varphi(t)\|_{X}<+\infty \quad \text { if } q=+\infty \text {. }
\end{aligned}
$$

We set

$$
\begin{gathered}
H_{q}=H_{q}(\Omega):={\overline{\left\{\boldsymbol{\varphi} \in C_{0}^{\infty}(\Omega) \mid \operatorname{div} \boldsymbol{\varphi}=0\right\}}}^{\|\cdot\|_{L}{ }^{q}(\Omega)}, \\
V_{q}=V_{q}(\Omega):={\overline{\left\{\boldsymbol{\varphi} \in C_{0}^{\infty}(\Omega) \mid \operatorname{div} \boldsymbol{\varphi}=0\right\}}}^{\|\cdot\|_{V_{q}}},
\end{gathered}
$$

where

$$
\|\boldsymbol{\varphi}\|_{V_{q}}:=\|\mathbf{D}(\boldsymbol{\varphi})\|_{L^{q}(\Omega)} .
$$


In the case that $q=2$ we omit the subscript $q$ and denote $H:=H_{2}$ and $V:=V_{2}$. It immediately follows from our definition of $W_{0}^{1, q}(\Omega)$ and $[39,18]$ that there exists a positive constant $\gamma_{0}$, such that the following Korn's inequality holds

$$
\|\nabla \mathbf{v}\|_{L^{q}(\Omega)} \leq \gamma_{0}\|\mathbf{D}(\mathbf{v})\|_{L^{q}(\Omega)} \quad \forall \mathbf{v} \in W_{0}^{1, q}(\Omega) .
$$

Definition 1.1. Assume that $\mathbf{S}$ satisfies (I) and (II) with $q \in\left[\frac{2 n}{n+2}, \infty\right)$. Let $\mathbf{F} \in$ $L^{1}\left(Q_{T}\right)$ and $\mathbf{u}_{0} \in H$. A vector-valued function $\mathbf{u} \in L^{q}\left(0, T ; V_{q}\right) \cap L^{\infty}(0, T ; H)$ is called a weak solution to (1.1)-(1.4) if the following identity

$$
\begin{aligned}
& -\int_{Q_{T}} \mathbf{u} \cdot \partial_{t} \varphi \mathrm{d} x \mathrm{~d} t+\int_{Q_{T}} \mathbf{S}(x, t, \mathbf{D}(\mathbf{u})): \mathbf{D}(\varphi) \mathrm{d} x \mathrm{~d} t \\
& -\int_{Q_{T}}(\mathbf{u} \otimes \mathbf{u}): \mathbf{D}(\varphi) \mathrm{d} x \mathrm{~d} t \\
= & \int_{Q_{T}} \mathbf{F}: \nabla \boldsymbol{\varphi} \mathrm{d} x \mathrm{~d} t+\int_{\Omega} \mathbf{u}_{0} \cdot \boldsymbol{\varphi}(0) \mathrm{d} x
\end{aligned}
$$

holds for all $\varphi \in C^{\infty}\left(Q_{T}\right)$ with $\operatorname{div} \varphi=0$ and $\operatorname{supp}(\varphi) \subset \subset \Omega \times[0, T)^{4}$.

Remark 1.2. By virtue of Sobolev's embedding theorem and Hölder's inequality one easily verifies

$$
L^{q}\left(0, T ; V_{q}\right) \cap L^{\infty}(0, T ; H) \hookrightarrow L^{q \frac{n+2}{n}}\left(Q_{T}\right) .
$$

The aim of the present paper is to prove the existence of a weak solution to the system (1.1)-(1.4) for such $q$ that $\operatorname{div}(\mathbf{u} \otimes \mathbf{u})$ merely belongs to the space $L^{1}(0, T$; $\left.\left(W_{0}^{1, \infty}(\Omega)\right)^{*}\right)$. Our main result is the following:

Theorem 1.3 (Main theorem). Let $\Omega \subset \mathbb{R}^{n}, n \geq 2$, be a bounded open set, and $0<T<\infty$. Assume that $\mathbf{S}$ satisfies (I), (II), (III), and (IV) for some $q$ with

$$
\frac{2 n}{n+2}<q<\infty
$$

and $\delta \geq 0$. Suppose that $\mathbf{u}_{0} \in H$ and $\mathbf{F} \in L^{q^{\prime}}\left(Q_{T}\right)$ are given. Then there exists a weak solution $\mathbf{u} \in L^{q}\left(0, T ; V_{q}\right) \cap C_{w}([0, T] ; H)$ to $(1.1)-(1.4)$.

\section{Remark 1.4.}

1. The mathematical analysis of the the system (1.1)-(1.3) was initiated by Ladyzhenskaya ( $c f .[25,27,28])$. She proved the existence of weak solutions in the case of Dirichlet boundary conditions (1.4) for $q \geq 1+\frac{2 n}{n+2}$ using the monotone operator theory combined with compactness arguments. This weak solution is unique if $q \geq \frac{n+2}{2}$ and $\mathbf{u}_{0} \in H$. The same results have been proved in [30] for the extra stress tensor $\mathbf{S}$ depending on the full velocity gradient $\nabla \mathbf{u}$ rather than on $\mathbf{D}(\mathbf{u})$ only.

${ }^{4}$ Here $A \subset \subset B$ means $A, B$ are open subsets of $\mathbb{R}^{n}, A$ is bounded and $\bar{A} \subset B$. 
2. The special case $q=2$, for which (1.1)-(1.2) are just the classical Navier-Stokes equations, cannot be treated with the theory of monotone operators if $n \geq 3$. However, the existence of weak solutions in the case of Dirichlet boundary conditions (1.4) is well known (cf. [29, 22]).

3. The existence of measure-valued solutions for the system(1.1)-(1.3) with Dirichlet boundary conditions (1.4) was proved for $q>\frac{2 n}{n+2}$ in [41, 21] (cf. [33, 32]) using Ball's theorem on Young measures. The existence of solutions satisfying a variational inequality instead of (1.8) was proved for the system (1.1)-(1.3) with Dirichlet boundary conditions (1.4) for $q \geq 2, n \geq 2$ in [8].

4. The lower bound for the existence of weak solutions of the system (1.1)-(1.3) with space periodic boundary conditions was extended to $q>\frac{3 n}{n+2}$ by Nečas and his collaborators $(c f .[33,5,32])$. The results have been obtained by using $-\Delta \mathbf{u}$ as a test function and deriving higher fractional differentiability in space. At the same time this technique gives the existence of a more regular solution, namely $\mathbf{u} \in L^{2}\left(0, T ; W^{2,2}(\Omega)\right) \cap L^{\infty}\left(0, T ; V_{q}\right) \cap L^{q}\left(0, T ; V_{\frac{q n}{n-2}}\right), \partial_{t} \mathbf{u} \in L^{2}\left(Q_{T}\right)$ for $q \geq 1+\frac{2 n}{n+2}$. This solution is unique in the class of weak solutions, provided $\mathbf{u}_{0} \in V_{2}$. The question of the uniqueness of weak solutions for values of $p$ below $1+\frac{2 n}{n+2}$ is still an open problem, which is not adressed in the paper. Note that the extra stress tensor $\mathbf{S}$ must satisfy slightly more restrictive assumptions than (I)-(IV), namely instead of (IV) one needs that $\mathbf{S}$ is uniformly monotone, i.e. $\frac{\partial \mathbf{S}(\boldsymbol{\xi})}{\partial \boldsymbol{\xi}}: \boldsymbol{\eta} \otimes \boldsymbol{\eta} \geq c(1+|\boldsymbol{\xi}|)^{q-2}|\boldsymbol{\eta}|^{2}$ holds for all $\boldsymbol{\xi}, \boldsymbol{\eta} \in \mathbb{M}_{\text {sym }}^{n}(c f$. [45,46]). The method was extended in [34] to Dirichlet boundary condition proving for $2 \leq$ $q<3, n=3$, the existence of weak solutions and some additional regularity properties for $\frac{9}{4} \leq q<3, n=3$ ( $c f$. [10,11] for some improvements). The special case $q=3, n=2,3$, which corresponds to the Smagorinsky model in turbulence, was treated in [42], proving the existence of a unique weak solution which additionally satisfies $\partial_{t} \mathbf{u} \in L^{2}\left(0, T ; V_{2}\right) \cap L^{\infty}(0, T ; H)$, provided $\mathbf{u}_{0} \in$ $H \cap W^{2,2}(\Omega)$. The existence of local in time strong solutions of the system (1.1)(1.3) with space periodic boundary conditions was proved in [32] for $q>\frac{5}{3}$, $n=3$. This result was improved in [14] to $q>\frac{7}{5}, n=3$, also providing better regularity properties of the solution. The case of Dirichlet boundary conditions was considered in [7]. In that paper $\mathbf{S}$ is assumed to be uniformly monotone with $q \geq 1$. The case of small data and global in time strong solutions has been treated in [2].

5. The lower bound for the existence of weak solutions of the system (1.1)-(1.3) with space periodic boundary conditions was further extended to $q>\frac{2(n+1)}{n+2}$ in [16] using $L^{\infty}$-test functions. The same result was obtained in [52] in the case of Dirichlet boundary conditions using $L^{\infty}$-test functions and the local pressure method.

6. The result in Theorem 1.3 is optimal in the following sense: For $q<\frac{2 n}{n+2}$ the term

$$
\int_{Q_{T}}(\mathbf{u} \otimes \mathbf{u}): \mathbf{D}(\boldsymbol{\varphi}) \mathrm{d} x \mathrm{~d} t, \quad \boldsymbol{\varphi} \in L^{\infty}\left(0, T ; W_{0}^{1, \infty}(\Omega)\right)
$$


is not well defined using the parabolic embedding (1.9) since $q \frac{n+2}{n}<2$. Nevertheless it is still possible to give sense to the above integral using the information $\mathbf{u} \in L^{\infty}\left(0, T ; L^{2}(\Omega)\right)$ coming from the time derivative only. Moreover, for $q<\frac{2 n}{n+2}$ the space $W_{0}^{1, q}(\Omega)$ does not embed into $L^{2}(\Omega)$. Thus we have that $L^{q}\left(0, T ; W_{0}^{1, q}(\Omega)\right) \hookrightarrow L^{q}\left(0, T ;\left(W_{0}^{1, q}(\Omega)\right)^{*}\right)$. Consequently, there are problems to give sense to the distributional time derivative.

The paper is organized as follows. In Section 2 we use the local pressure method to introduce the pressure for the problem (1.1), (1.2) and decompose it similar to the procedure in [52]. However, here we use only standard results for the Stokes system and the divergence equation. In Section 3 we establish the Lipschitz truncation method for unsteady problems. For that we use a Whitney type decomposition to extend a function in which irregular regions have been cut off before. We also prove that this extension belongs to the space $L^{\infty}\left(0, T ; W^{1, \infty}(\Omega)\right)$ and that a certain partial integration formula with respect to time holds true. Similar results have been proved in [24]. Here it is to the knowledge of the authors the first time that the Lipschitz truncation method is used to prove an existence result for unsteady problems. For steady problems the Lipschitz truncation method was introduced in [1]. In Section 4 we approximate the problem (1.1), (1.2) appropriately. The approximate problem possesses weak solutions ( $c f$. [52]) for which we justify the limiting process using essentially the properties of the Lipschitz truncation established before. This gives a proof of the main Theorem 1.3. The steady version of the problem $(1.1),(1.2)$ was treated in $[17,13]$ with the help of the Lipschitz truncation method.

\section{Pressure representation}

The aim of this section is to introduce the pressure for the problem (1.1)-(1.2). We decompose the pressure corresponding to the respective terms appearing in the equation. This forms the basis for studying the local behaviour of the velocity and the pressure.

Throughout this section let $G \subset \mathbb{R}^{n}$ be a bounded domain with $\partial G \in C^{2}$.

Lemma 2.1. Let $1<s<\infty$. Let $\mathbf{v}^{*} \in\left(W_{0}^{1, s}(G)\right)^{*}$ with $\left\langle\mathbf{v}^{*}, \mathbf{v}\right\rangle=0$ for all $\mathbf{v} \in V_{s}(G)$. Then there exists a unique $p \in L^{s}(G)$ with $p_{G}=0$, such that

$$
\left\langle\mathbf{v}^{*}, \mathbf{v}\right\rangle=\int_{G} p \operatorname{div} \mathbf{v} \mathrm{d} x \quad \forall \mathbf{v} \in W_{0}^{1, s}(G) .
$$

Furthermore there holds

$$
\|p\|_{L^{s}(G)} \leq c\left\|\mathbf{v}^{*}\right\|_{\left(W_{0}^{1, s}(G)\right)^{*}}
$$

Proof. [19, Theorems III.3.1 and III.5.2].

Arguing similar as in [52] we will introduce the pressure. 
Theorem 2.2. Let $\mathbf{u} \in C_{w}([0, T] ; H(G))$ and let $\mathbf{H}_{\mathbf{i}} \in L^{s_{i}}\left(0, T ; L^{s_{i}}(G)\right)$, $1<s_{i}<\infty, i=1,2$. Suppose that

$$
-\int_{0}^{T} \int_{G} \mathbf{u} \cdot \partial_{t} \boldsymbol{\varphi} \mathrm{d} x \mathrm{~d} t=\int_{0}^{T} \int_{G}\left(\mathbf{H}_{1}+\mathbf{H}_{2}\right): \nabla \boldsymbol{\varphi} \mathrm{d} x \mathrm{~d} t
$$

holds for all $\boldsymbol{\varphi} \in C_{0}^{\infty}(G \times(0, T))$ with $\operatorname{div} \varphi=0$. Then there exist unique functions $p_{i} \in L^{s_{i}}\left(0, T ; L^{s_{i}}(G)\right), i=1,2$, and $p_{h} \in C_{w}\left([0, T] ; W^{1,2}(G)\right)$, such that

$$
\begin{aligned}
-\int_{0}^{T} \int_{G} \mathbf{u} \cdot \partial_{t} \boldsymbol{\varphi} \mathrm{d} x \mathrm{~d} t= & \int_{0}^{T} \int_{G}\left(\mathbf{H}_{1}+\mathbf{H}_{2}\right): \nabla \boldsymbol{\varphi} \mathrm{d} x \mathrm{~d} t \\
& +\int_{0}^{T} \int_{G}\left(p_{1}+p_{2}\right) \mathrm{div} \boldsymbol{\varphi}+\int_{0}^{T} \int_{G} \nabla p_{h} \cdot \partial_{t} \boldsymbol{\varphi} \mathrm{d} x \mathrm{~d} t \\
& +\int_{G} \mathbf{u}(0) \cdot \boldsymbol{\varphi}(0) \mathrm{d} x
\end{aligned}
$$

for all $\varphi \in C^{\infty}(G \times(0, T))$ with $\operatorname{supp}(\varphi) \subset \subset G \times[0, T)$. In addition, we have $-\Delta p_{h}=0, p_{h}(0)=0$ and the apriori estimates

$$
\begin{aligned}
\left\|p_{i}\right\|_{L^{s_{i}(G \times(0, T))}} & \leq c_{i}\left\|\mathbf{H}_{i}\right\|_{L^{s_{i}(G \times(0, T))},} & & i=1,2, \\
\left\|p_{h}(t)\right\|_{W^{1,2}(G)} & \leq c_{h}\|\mathbf{u}(t)-\mathbf{u}(0)\|_{L^{2}(G)}, & & t \in[0, T],
\end{aligned}
$$

with constants $c_{i}$ depending only on $n, G$, and $s_{i}, i=1,2$, and a constant $c_{h}$ depending only on $n$ and $G$.

Proof. Arguing as in the proof of [52, Theorem 2.6] one gets the existence of a pressure $\tilde{p} \in C_{w}\left([0, T] ; L^{1}(G)\right)$, such that

$$
\begin{aligned}
\int_{G}(\mathbf{u}(t)-\mathbf{u}(0)) \boldsymbol{\psi} \mathrm{d} x= & \int_{G}\left(\widetilde{\mathbf{H}}_{1}(t)+\widetilde{\mathbf{H}}_{2}(t)\right): \nabla \boldsymbol{\psi} \mathrm{d} x \\
& +\int_{G} \tilde{p}(t) \operatorname{div} \boldsymbol{\psi} \mathrm{d} x, \quad \forall \psi \in W_{0}^{1,2}(G),
\end{aligned}
$$

for all $0 \leq t \leq T$, where

$$
\widetilde{\mathbf{H}}_{i}(t)=\int_{0}^{t} \mathbf{H}_{i}(\tau) \mathrm{d} \tau, \quad 0<t<T, \quad i=1,2 .
$$

Without loss of generality we may assume that $\tilde{p}(t)_{G}=0$ for all $0 \leq t \leq T$. Note that in this case the pressure $\tilde{p}$ is unique. problem

Next, let $\mathbf{v}_{i}(t) \in V_{s_{i}}(G), 0 \leq t \leq T$, denote the unique solution to the Stokes

$$
\begin{aligned}
-\Delta \mathbf{v}_{i}(t)+\nabla \pi_{i}(t) & =-\operatorname{div} \widetilde{\mathbf{H}}_{i}(t) & & \text { in } G, \\
\operatorname{div} \mathbf{v}_{i}(t) & =0 & & \text { in } G, \\
\mathbf{v}_{i}(t) & =0 & & \text { on } \partial G,
\end{aligned}
$$


for $i=1$, 2. According to Lemma 2.1 we have $\pi_{i}(t) \in L^{s_{i}}(G)$ with $\left(\pi_{i}(t)\right)_{G}=0$. Replacing in (2.7) $t$ by $t+h, 0<h \leq T-h$, and taking the difference of both equations we get in view of Lemma 2.1 the estimate

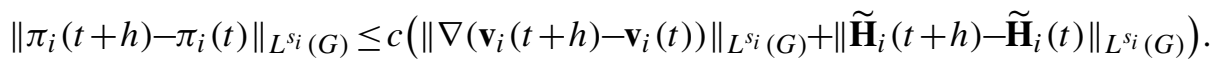

From [20, Theorem 2.1], and [19, Theorem IV.6.1] we obtain

$$
\left\|\nabla\left(\mathbf{v}_{i}(t+h)-\mathbf{v}_{i}(t)\right)\right\|_{L^{s_{i}}(G)} \leq c\left\|\widetilde{\mathbf{H}}_{i}(t+h)-\widetilde{\mathbf{H}}_{i}(t)\right\|_{L^{s_{i}}(G)},
$$

which leads to

$$
\left\|\pi_{i}(t+h)-\pi_{i}(t)\right\|_{L^{s_{i}(G)}}^{s_{i}} \leq c\left\|\int_{t}^{t+h} \mathbf{H}_{i}(\tau) \mathrm{d} \tau\right\|_{L^{s_{i}(G)}}^{s_{i}} .
$$

Dividing both sides by $h^{s_{i}}$, integrating over the interval $(0, T-h)$, applying Hölder's inequality and Fubini's theorem yields

$$
\int_{0}^{T-h} \frac{\left\|\pi_{i}(t+h)-\pi_{i}(t)\right\|_{L^{s_{i}}(G)}^{s_{s_{i}}}}{h^{s_{i}}} \mathrm{~d} t \leq c\left\|\mathbf{H}_{i}\right\|_{L^{s_{i}}(G \times(0, T))}^{s_{i}} .
$$

This shows that $\pi_{i} \in W^{1, s_{i}}\left(0, T ; L^{s_{i}}(G)\right)$. Set $p_{i}:=\partial_{t} \pi_{i}$. In the estimate above passing to the limit as $h \rightarrow 0$ implies (2.4)

Next, let $\mathbf{v}_{h}(t) \in V_{2}(G) \cap W^{2,2}(G), 0 \leq t \leq T$, denote the unique solution to the Stokes problem

$$
\begin{aligned}
-\Delta \mathbf{v}_{h}(t)+\nabla p_{h}(t) & =-\mathbf{u}(t)+\mathbf{u}(0) & & \text { in } G, \\
\operatorname{div} \mathbf{v}_{h}(t) & =0, & & \text { in } G, \\
\mathbf{v}_{h}(t) & =0 & & \text { on } \partial G .
\end{aligned}
$$

Appealing to [19, Theorem IV.6.1] one finds $p_{h}(t) \in W^{1,2}(G)$ with $\left(p_{h}(t)\right)_{G}=0$. Moreover, we have

$$
\left\|p_{h}(t)\right\|_{W^{1,2}(G)} \leq c\|\mathbf{u}(t)-\mathbf{u}(0)\|_{L^{2}(G)}
$$

Whence, (2.5).

Define $\mathbf{v}(t):=\mathbf{v}_{1}(t)+\mathbf{v}_{2}(t)+\mathbf{v}_{h}(t)$. From (2.6) follows

$$
-\operatorname{div} \widetilde{\mathbf{H}}_{2}(t)-\operatorname{div} \widetilde{\mathbf{H}}_{2}(t)-\mathbf{u}(t)+\mathbf{u}(0)=\nabla \tilde{p}(t) .
$$

Thus, taking the sum of (2.7) and (2.8) leads to

$$
\begin{aligned}
-\Delta \mathbf{v}(t)+\nabla\left(\pi_{1}(t)+\pi_{2}(t)+p_{h}(t)-\tilde{p}(t)\right)=\mathbf{0} & \text { in } G, \\
\operatorname{div} \mathbf{v}(t)=0, & \text { in } G, \\
\mathbf{v}(t)=\mathbf{0} & \text { on } \partial G,
\end{aligned}
$$

which implies $\mathbf{v}=\mathbf{0}$ and $\tilde{p}(t)=\pi_{1}(t)+\pi_{2}(t)+p_{h}(t)$. Thus, inserting $\boldsymbol{\psi}(\cdot, t)=$ $\partial_{t} \varphi(\cdot, t), t \in(0, T)$, into (2.6), integrating this identity over $(0, T)$, and applying integration by parts yields (2.3). 


\section{Parabolic Lipschitz truncation}

In this section we will establish a Lipschitz truncation method for unsteady problems. The idea is to regularize a given function by cutting off regions of irregularity and then to extend this restricted function by a Whitney type extension to the whole domain again. In a different context this method has been used in [24]. The basis of this approach is a covering lemma of Whitney type, which is well-known (cf. [48, Chapter 6], [12, Chapter1], [9, Theorem 3.2]). Here we use these general results for the Euclidean space $\mathbb{R}^{n+1}$ with an anisotropic parabolic metric. The typical points $(x, t)$ and $(y, s)$ of $\mathbb{R}^{n+1}$ are shortly denoted by $X$ and $Y$, respectively. Given $\alpha>0$ we equip $\mathbb{R}^{n+1}$ with the following anisotropic (scaled parabolic) metric

$$
d_{\alpha}(X, Y):=\max \left\{|x-y|,\left|\alpha^{-1}(s-t)\right|^{1 / 2}\right\}, \quad X, Y \in \mathbb{R}^{n+1} .
$$

For $X_{0} \in \mathbb{R}^{n+1}$ and $r>0$ we define balls $Q_{r}^{\alpha}\left(X_{0}\right):=\left\{X \in \mathbb{R}^{n+1} \mid d_{\alpha}\left(X, X_{0}\right)<r\right\}$, which are equal to the cylindrical sets $B_{r}\left(x_{0}\right) \times\left(t_{0}-\alpha r^{2}, t_{0}+\alpha r^{2}\right)$. One easily checks that for all $X \in \mathbb{R}^{n+1}, 0<r<\infty$ and $\alpha>0$ holds

$$
\mathcal{L}_{n+1}\left(Q_{r}^{\alpha}(X)\right) \leq 2^{n+2} \mathcal{L}_{n+1}\left(Q_{\frac{r}{2}}^{\alpha}(X)\right)
$$

This implies that $\left(\mathbb{R}^{n+1}, d_{\alpha}\right)$ satisfies the following homogeneity property:

There exists a number $\hat{N} \in \mathbb{N}$, depending only on $n$, such that, each cylinder $Q_{r}\left(X_{0}\right)$ contains no more than $\hat{N}$ points $\left\{X_{i}\right\}$ with $d_{\alpha}\left(X_{i}, X_{j}\right) \geq \frac{r}{4}$ for $i \neq j$.

Thus we have ( $c f$. [48, Chapter 6], [12, Chapter 1], [9, 47, Theorem 3.2]) the following result:

Lemma 3.1 (Whitney type covering). Let $E$ be a non-empty, open, bounded set of $\mathbb{R}^{n+1}$ equipped with the metric $d_{\alpha}, \alpha>0$. Then there exists a covering $\left\{Q_{i}^{\alpha}\right\}=$ $\left\{Q_{r_{i}^{\alpha}}^{\alpha}\left(X_{i}^{\alpha}\right)\right\}$ of $E$, such that ${ }^{5}$

(W1) $\bigcup_{i} \frac{1}{2} Q_{i}^{\alpha}=E$,

(W2) for all $i \in \mathbb{N}$ we have $8 Q_{i}^{\alpha} \subset E$ and $16 Q_{i}^{\alpha} \cap\left(\mathbb{R}^{n+1} \backslash E\right) \neq \emptyset$,

(W3) if $Q_{i}^{\alpha} \cap Q_{j}^{\alpha} \neq \emptyset$ then $\frac{1}{2} r_{j}^{\alpha} \leq r_{i}^{\alpha}<2 r_{j}^{\alpha}$,

(W4) each $X \in E$ belongs to at most $120^{n+2}$ of the sets $4 Q_{i}^{\alpha}$.

We have denoted by $\gamma Q_{i}^{\alpha}(\gamma>0)$ the ball $Q_{\gamma r_{i}^{\alpha}}^{\alpha}\left(X_{i}^{\alpha}\right)$. Note that this scaling depends on $\alpha$.

For the readers convenience an elementary proof can be found in the Appendix $\mathrm{C}$ of this paper.

${ }^{5}$ By $\gamma Q_{i}^{\alpha}(\gamma>0)$ we denote the ball $Q_{\gamma r_{i}^{\alpha}}^{\alpha}\left(X_{i}^{\alpha}\right)$. 


\section{Remark 3.2.}

1. The covering $\left\{Q_{i}^{\alpha}\right\}$ of $E$ has the following additional properties:

$$
\sum_{j} \mathcal{L}_{n+1}\left(4 Q_{j}^{\alpha}\right) \leq 120^{n+2} \mathcal{L}_{n+1}(E)
$$

Property (W4) of the above lemma can be written as $\sum_{i} \chi_{Q_{i}^{\alpha}} \leq 120^{n+2}$, which implies

$$
\sum_{j} \mathcal{L}_{n+1}\left(4 Q_{j}^{\alpha}\right)=\sum_{j} \int_{E} \chi_{4 Q_{j}^{\alpha}} \mathrm{d} X \leq 120^{n+2} \mathcal{L}_{n+1}(E) .
$$

2. Define $A_{i}:=\left\{j \in \mathbb{N} \mid \frac{2}{3} Q_{j}^{\alpha} \cap \frac{2}{3} Q_{i}^{\alpha} \neq \emptyset\right\}(i=1,2, \ldots)$. Note that from Lemma C. 1 follows that $\# A_{i} \leq 120^{n+2}$. Moreover, we have

$$
Q_{j}^{\alpha} \subset 4 Q_{i}^{\alpha} \subset E \quad \text { for all } j \in A_{i} \text {. }
$$

Indeed, if $\frac{2}{3} Q_{j}^{\alpha} \cap \frac{2}{3} Q_{i}^{\alpha} \neq \emptyset$, then we have $r_{j}^{\alpha} \leq 2 r_{i}^{\alpha}$ due to (W3). For $Y \in Q_{j}^{\alpha}$ we estimate

$$
\begin{aligned}
d_{\alpha}\left(X_{i}^{\alpha}, Y\right) & \leq d_{\alpha}\left(X_{i}^{\alpha}, X\right)+d_{\alpha}\left(X, X_{j}^{\alpha}\right)+d_{\alpha}\left(X_{j}^{\alpha}, Y\right) \\
& <\frac{2}{3} r_{i}^{\alpha}+\frac{4}{3} r_{i}^{\alpha}+2 r_{i}^{\alpha}=4 r_{i}^{\alpha} .
\end{aligned}
$$

Consequently, $Y \in 4 Q_{i}^{\alpha}$.

Next, let us introduce the notion of Lipschitz continuous functions with respect to the metric $d_{\alpha}$.

Definition 3.3. For a given Lebesgue measurable set $A \subset \mathbb{R}^{n+1}$ by $C_{d_{\alpha}}^{0,1}(A)$ we denote the space of all essentially bounded functions $u: A \rightarrow \mathbb{R}$, satisfying

$$
\operatorname{Lip}_{d_{\alpha} ; A}(u)<\infty
$$

where

$$
\operatorname{Lip}_{d_{\alpha} ; A}(u):=\sup _{\substack{X, Y \in A \\ X \neq Y}} \frac{|u(X)-u(Y)|}{d_{\alpha}(X, Y)}
$$

Partition of unity. Let $E$ be a non-empty, bounded, open subset of $\mathbb{R}^{n+1}$ equipped with the metric $d_{\alpha}$. Let $\left\{Q_{i}^{\alpha}\right\}$ denote a Whitney type covering of $E$ from Lemma 3.1. Let $\eta_{i} \in C_{0}^{\infty}\left(\mathbb{R}^{n+1}\right)$ denote cut-off functions with $0 \leq \eta_{i} \leq 1$ in $\mathbb{R}^{n+1}, \eta_{i}=0$ in $\mathbb{R}^{n+1} \backslash \frac{2}{3} Q_{i}^{\alpha}, \eta_{i}=1$ on $\frac{1}{2} Q_{i}^{\alpha}$, such that

$$
\operatorname{Lip}_{d_{\alpha}}\left(\eta_{i}\right)+\left|\alpha \partial_{t} \eta_{i}\right|^{\frac{1}{2}} \leq c_{n}\left(r_{i}^{\alpha}\right)^{-1} \quad \text { in } \quad \mathbb{R}^{n+1} \quad(i \in \mathbb{N})
$$


Recalling the definition of $A_{i}$ (cf. Remark 3.2) we have

$$
\sum_{j} \eta_{j}=\sum_{j \in A_{i}} \eta_{j} \quad \text { in } \quad Q_{i}^{\alpha}
$$

and $\sum_{j} \eta_{j} \in C^{\infty}\left(\mathbb{R}^{n+1}\right)$. Next, we set

$$
\psi_{i}(X):=\frac{\eta_{i}(X)}{\sum_{j} \eta_{j}(X)}, \quad X \in E \quad(i \in \mathbb{N})
$$

which is well defined, since by (W1) there holds $\sum_{j} \eta_{j} \geq 1$ in $E$. Clearly, we have that $\psi_{i} \in C^{\infty}\left(\mathbb{R}^{n+1}\right)$ satisfy $\psi_{i} \equiv 0$ in $\mathbb{R}^{n+1} \backslash \frac{2}{3} Q_{i}^{\alpha}$. Moreover, we have

$$
\sum_{j} \psi_{j}=\sum_{j \in A_{i}} \psi_{j}=1 \quad \text { in } Q_{i}^{\alpha}
$$

Properties (W1), (W2), (W3), (W4), and (3.1) yield

$$
\begin{aligned}
\left|\psi_{i}(X)-\psi_{i}(Y)\right| & \leq\left|\eta_{i}(X)-\eta_{i}(Y)\right|+\sum_{j \in A_{i}}\left|\eta_{j}(X)-\eta_{j}(Y)\right| \\
& \leq c_{n}\left(\left(r_{i}^{\alpha}\right)^{-1}+\sum_{j \in A_{i}}\left(r_{j}^{\alpha}\right)^{-1}\right) d_{\alpha}(X, Y) \\
& \leq c_{n}\left(1+2 \cdot 120^{n+2}\right)\left(r_{i}^{\alpha}\right)^{-1} d_{\alpha}(X, Y) .
\end{aligned}
$$

Similarly, one proves that

$$
\left|\partial_{t} \psi_{i}\right| \leq\left|\partial_{t} \eta_{i}\right|+\sum_{j \in A_{i}}\left|\partial_{t} \eta_{j}\right| \leq c_{n}^{2}\left(1+4 \cdot 120^{n+2}\right) \alpha^{-1}\left(r_{i}^{\alpha}\right)^{-2} \quad \text { in } \quad \mathbb{R}^{n+1} .
$$

Thus,

$$
\operatorname{Lip}_{d_{\alpha}}\left(\psi_{i}\right)+\left|\alpha \partial_{t} \psi_{i}\right|^{\frac{1}{2}} \leq c_{n}\left(1+4 \cdot 120^{n+2}\right)\left(r_{i}^{\alpha}\right)^{-1} \text { in } \mathbb{R}^{n+1} .
$$

Definition of the truncation operator. Let $G \subset \mathbb{R}^{n}$ be a non-empty, open, bounded set and $0<T<\infty$. For a non-empty, open set $E \subset G \times(0, T)$ let $\left\{Q_{i}^{\alpha}\right\}$ be the corresponding Whitney covering from Lemma 3.1 for the metric $d_{\alpha}$, and $\left\{\psi_{i}\right\}$ the associated partition of unity. For $u \in L_{\text {loc }}^{1}(G \times(0, T))$ we define

$$
\left(\mathcal{T}_{E}^{\alpha} u\right)(X):= \begin{cases}u(X) & \text { if } \quad X \in(G \times(0, T)) \backslash E, \\ \sum_{i=1}^{\infty} \psi_{i}(X) u_{Q_{i}^{\alpha}} & \text { if } \quad X \in E .\end{cases}
$$

From the construction of $\mathcal{T}_{E}^{\alpha} u$ it is clear that the restriction of $\mathcal{T}_{E}^{\alpha} u$ upon $E$ is smooth.

Next, we prove several properties of the operator $\mathcal{T}_{E}^{\alpha} u$, which will be used in what follows. 
Lemma 3.4. Let $u \in L_{\mathrm{loc}}^{1}(G \times(0, T))$. Then there holds

$$
\left|\mathcal{T}_{E}^{\alpha} u\right| \leq 10^{n+2} \mathcal{M}^{*}(|u|) \text { a.e. in } G \times(0, T),
$$

where $c=$ const $>0$.

Proof. If $X \in(G \times(0, T)) \backslash E$, then $\left|T_{E}^{\alpha} u(X)\right|=|u(X)| \leq \mathcal{M}^{*}(|u|)(X)$ by the properties of $\mathcal{M}^{*}$ (cf. Appendix A). Now, let $X \in E$. Then by (W1) we have $X \in Q_{i}^{\alpha}$ for some $i$. By the definition of $\mathcal{T}_{E}^{\alpha} u$ and of the set $A_{i}$ it follows that

$$
\left(\mathcal{T}_{E}^{\alpha} u\right)(X)=\sum_{j \in A_{i}} u_{Q_{j}^{\alpha}} \psi_{j}(X) .
$$

Taking into account (W6) and (W3) implies

$$
\left|\mathcal{T}_{E}^{\alpha} u(X)\right| \leq \sum_{j \in A_{i}}\left|u_{Q_{j}^{\alpha}}\right| \psi_{j}(X) \leq 8^{n+2} f_{4 Q_{i}^{\alpha}}|u| \mathrm{d} Y .
$$

Since $4 Q_{i}^{\alpha} \subset Q_{5 r_{i}^{\alpha}}^{\alpha}(X)$ we obtain

$$
\left|\mathcal{T}_{E}^{\alpha} u(X)\right| \leq 10^{n+2} f_{Q_{S_{i}^{\alpha}}^{\alpha}(X)}|u| \mathrm{d} Y \leq 10^{n+2} \mathcal{M}^{*}(|u|)(X) .
$$

The proof of the lemma is completed.

Lemma 3.5. For every $1 \leq p \leq \infty$ there exists a constant $c$ such that

$$
\left\|\mathcal{T}_{E}^{\alpha} u\right\|_{L^{p}(G \times(0, T))} \leq c\|u\|_{L^{p}(G \times(0, T))} \quad \forall u \in L^{p}(G \times(0, T)),
$$

where $c$ depends only $n$.

Proof. By the definition of $\mathcal{T}_{E}^{\alpha}$ and the properties (W1), (W6), and (W5) of the Whitney covering we have

$$
\begin{aligned}
\int_{G \times(0, T)}\left|\left(\mathcal{T}_{E}^{\alpha} u\right)(X)\right| \mathrm{d} X & =\int_{G \times(0, T) \backslash E}|u(X)| \mathrm{d} X+\int_{E}\left|\left(\mathcal{T}_{E} u\right)(X)\right| \mathrm{d} X \\
& \leq\|u\|_{L^{1}(G \times(0, T))}+\sum_{i} \int_{Q_{i}^{\alpha}}\left|\left(\mathcal{T}_{E} u\right)(X)\right| \mathrm{d} X \\
& \leq\|u\|_{L^{1}(G \times(0, T))}+\sum_{i} \int_{Q_{i}^{\alpha}}\left(\sum_{j \in A_{i}} f_{Q_{j}}|u(Y)| \mathrm{d} Y\right) \mathrm{d} X \\
& \leq\|u\|_{L^{1}(G \times(0, T))}+2^{n+2} \sum_{i} \sum_{j \in A_{i}} \int_{4 Q_{i}^{\alpha}}|u(X)| \mathrm{d} X \\
& \leq\left(1+240^{n+2}\right)\|u\|_{L^{1}(G \times(0, T))} .
\end{aligned}
$$


From the definition of $\mathcal{T}_{E}^{\alpha}$ follows

$$
\left\|\mathcal{T}_{E}^{\alpha} u\right\|_{L^{\infty}(G \times(0, T))} \leq\|u\|_{L^{\infty}(G \times(0, T))} .
$$

The assertion thus follows from interpolation also for $1<p<\infty$.

Lemma 3.6. Let $u \in L_{\mathrm{loc}}^{1}(G \times(0, T))$. Then we have for all $Y, Z \in Q_{i}^{\alpha}, i \in \mathbb{N}$

$$
\left|\mathcal{T}_{E}^{\alpha} u(Y)-\mathcal{T}_{E}^{\alpha} u(Z)\right| \leq c\left(r_{i}^{\alpha}\right)^{-1} d_{\alpha}(Y, Z) f_{4 Q_{i}^{\alpha}}\left|u-u_{4 Q_{i}^{\alpha}}\right| \mathrm{d} X,
$$

where $c$ depends only on $n$.

Proof. By the definition of $\mathcal{T}_{E}^{\alpha}$ one easily calculates for all $Y, Z \in Q_{i}^{\alpha}$

$$
\begin{aligned}
\mathcal{T}_{E}^{\alpha} u(Y)-\mathcal{T}_{E}^{\alpha} u(Z) & =\sum_{j \in A_{i}} u_{Q_{j}^{\alpha}}\left(\psi_{j}(Y)-\psi_{j}(Z)\right) \\
& =\sum_{j \in A_{i}}\left(u_{Q_{j}^{\alpha}}-u_{Q_{i}^{\alpha}}\right)\left(\psi_{j}(Y)-\psi_{j}(Z)\right),
\end{aligned}
$$

where we used (3.2). Using Jensen's inequality along with property (W6) one gets

$$
\left|u_{Q_{j}^{\alpha}}-u_{Q_{i}^{\alpha}}\right| \leq 4^{n+2} \cdot 2 f_{4 Q_{i}^{\alpha}}\left|u-u_{4 Q_{i}^{\alpha}}\right| \mathrm{d} X \quad \forall j \in A_{i} .
$$

The last two estimates together with the Lipschitz bound (3.4) and the properties (W3) and (W4) of the Whitney covering imply (3.8).

Lemma 3.7. Let $u \in L_{\mathrm{loc}}^{1}(G \times(0, T))$. Then for all $Y \in Q_{i}^{\alpha}, i \in \mathbb{N}$

$$
\left|\mathcal{T}_{E}^{\alpha} u(Y)-u(Y)\right| \leq c f_{4 Q_{i}^{\alpha}}|u(X)-u(Y)| \mathrm{d} X,
$$

where $c$ depends only on $n$.

Proof. Let $Y \in Q_{i}^{\alpha}, i \in \mathbb{N}$. The definition of $\mathcal{T}_{E}^{\alpha}$ and (W6) yield

$$
\left|\mathcal{T}_{E}^{\alpha} u(Y)-u(Y)\right| \leq \sum_{j \in A_{i}} \psi_{j}(Y)\left|u_{Q_{j}^{\alpha}}-u(Y)\right| \leq c f_{4 Q_{i}^{\alpha}}|u(X)-u(Y)| \mathrm{d} X .
$$

Whence (3.10).

Note that from Lemma 3.7 one obtains the following estimate for $i \in \mathbb{N}$

$$
f_{Q_{i}^{\alpha}}\left|\mathcal{T}_{E}^{\alpha} u-u\right| \mathrm{d} Y \leq c f_{4 Q_{i}^{\alpha}}\left|u-u_{4 Q_{i}^{\alpha}}\right| \mathrm{d} Y,
$$

where $c$ depends only on $n$. 
Lemma 3.8. Let $u \in L_{\mathrm{loc}}^{1}(G \times(0, T))$. Then for all $Y \in Q_{i}^{\alpha}, i \in \mathbb{N}$

$$
\left|\partial_{t} \mathcal{T}_{E}^{\alpha} u(Y)\right| \leq c \alpha^{-1}\left(r_{i}^{\alpha}\right)^{-2} f_{4 Q_{i}^{\alpha}}\left|u-u_{4 Q_{i}^{\alpha}}\right| \mathrm{d} X
$$

where c depends only on $n$.

Proof. Let $Y \in Q_{i}^{\alpha}, i \in \mathbb{N}$. By the definition of $\mathcal{T}_{E}^{\alpha}$ and (3.2) one gets

$$
\partial_{t} \mathcal{T}_{E}^{\alpha} u(Y)=\sum_{j \in A_{i}} \partial_{t}\left(\psi_{j}(Y)\left(u_{Q_{j}^{\alpha}}-u_{Q_{i}^{\alpha}}\right)\right)=\sum_{j \in A_{i}} \partial_{t} \psi_{j}(Y)\left(u_{Q_{j}^{\alpha}}-u_{Q_{i}^{\alpha}} .\right.
$$

Using (3.4), (3.9), (W3) and (W6) one estimates

$$
\left|\partial_{t} \mathcal{T}_{E}^{\alpha} u(Y)\right| \leq c \alpha^{-1}\left(r_{i}^{\alpha}\right)^{-2} \sum_{j \in A_{i}}\left|u_{Q_{j}^{\alpha}}-u_{Q_{i}^{\alpha}}\right| \leq c \alpha^{-1}\left(r_{i}^{\alpha}\right)^{-2} f_{4 Q_{i}^{\alpha}}\left|u-u_{4 Q_{i}^{\alpha}}\right| \mathrm{d} X
$$

This completes the proof of the lemma.

Now, we present the main result of this section, where we establish a formula of integration by parts with respect to the time derivative. This result plays an essential role in the proof of the main result given in Section 4.

Theorem 3.9. Let $\mathbf{u} \in L^{\infty}\left(0, T ; L^{2}(G)\right) \cap L^{q}\left(0, T ; W^{1, q}(G)\right)(1<q<\infty)$ and $\mathbf{H} \in L^{\sigma}\left(0, T ; L^{\sigma}(G)\right)(1<\sigma<\infty)$ be such that

$$
-\int_{G \times(0, T)} \mathbf{u} \cdot \partial_{t} \boldsymbol{\varphi} \mathrm{d} X=\int_{G \times(0, T)} \mathbf{H}: \nabla \boldsymbol{\varphi} \mathrm{d} X
$$

for all $\varphi \in C_{0}^{\infty}(G \times(0, T))$. We define

$$
\begin{aligned}
\mathcal{O}_{\Lambda} & :=\left\{X \in \mathbb{R}^{n+1} \mid \mathcal{M}^{*}(|\nabla \mathbf{u}|)(X)+\alpha \mathcal{M}^{*}(|\mathbf{H}|)(X)>\Lambda\right\}, \quad \Lambda>0 \\
\mathcal{U}_{1} & :=\left\{X \in \mathbb{R}^{n+1} \mid \mathcal{M}^{*}(|\mathbf{u}|)(X)>1\right\}
\end{aligned}
$$

Let $\Lambda>0$ and the open set $E \subset \mathbb{R}^{n+1}$ with $\mathcal{L}_{n+1}(E)<\infty$ be such that

$$
(G \times(0, T)) \cap\left(\mathcal{O}_{\Lambda} \cup \mathcal{U}_{1}\right) \subset E \subset G \times(0, T)
$$

Let $K \subset G \times(0, T)$ be a compact set. Then we have:

(i) The Lipschitz truncation $\mathcal{T}_{E}^{\alpha} \mathbf{u}$ belongs to $C_{d_{\alpha}}^{0,1}(K)$ with a norm depending on $n, K, \Lambda, \alpha,\|\mathbf{u}\|_{L^{1}(E)},\|\mathbf{u}\|_{L^{1}(\tilde{K} \times(0, T))}$, where the $K \subset \subset \tilde{K} \subset \subset G$. In particular, we have $\mathcal{T}_{E}^{\alpha} \mathbf{u}, \nabla \mathcal{T}_{E}^{\alpha} \mathbf{u} \in L^{\infty}(K)$. 
(ii) The Lipschitz truncation $\mathcal{T}_{E}^{\alpha} \mathbf{u}$ satisfies the estimates

$$
\begin{gathered}
\left\|\nabla \mathcal{T}_{E}^{\alpha} \mathbf{u}\right\|_{L^{\infty}(K)} \leq c\left(\Lambda+\alpha^{-1} \delta_{\alpha, K}^{-n-3}\|\mathbf{u}\|_{L^{1}(E)}\right), \\
\left\|\mathcal{T}_{E}^{\alpha} \mathbf{u}\right\|_{L^{\infty}(K)} \leq c\left(1+\alpha^{-1} \delta_{\alpha, K}^{-n-2}\|\mathbf{u}\|_{L^{1}(E)}\right),
\end{gathered}
$$

where $\delta_{\alpha, K}:=d_{\alpha}(K, \partial(G \times(0, T)))$ and where the constants $c$ depend only on $n$.

(iii) The function $\left(\partial_{t} \mathcal{T}_{E}^{\alpha} \mathbf{u}\right) \cdot\left(\mathcal{T}_{E}^{\alpha} \mathbf{u}-\mathbf{u}\right)$ belongs to $L^{1}(K \cap E)$ and we have

$$
\begin{aligned}
& \left\|\left(\partial_{t} \mathcal{T}_{E}^{\alpha} \mathbf{u}\right) \cdot\left(\mathcal{T}_{E}^{\alpha} \mathbf{u}-\mathbf{u}\right)\right\|_{L^{1}(K \cap E)} \\
& \leq c \alpha^{-1} \mathcal{L}_{n+1}(E)\left(\Lambda+\alpha^{-1} \delta_{\alpha, K}^{-n-3}\|\mathbf{u}\|_{L^{1}(E)}\right)^{2},
\end{aligned}
$$

where the constant $c$ depends only on $n$.

(iv) For all $\zeta \in C_{0}^{\infty}(G \times(0, T))$ holds the identity

$$
\begin{aligned}
\int_{0}^{T}\left\langle\partial_{t} \mathbf{u}(t),\left(\mathcal{T}_{E}^{\alpha} \mathbf{u}(t)\right) \zeta(t)\right\rangle \mathrm{d} t= & \frac{1}{2} \int_{G \times(0, T)}\left(\left|\mathcal{T}_{E}^{\alpha} \mathbf{u}\right|^{2}-2 \mathbf{u} \cdot \mathcal{T}_{E}^{\alpha} \mathbf{u}\right) \partial_{t} \zeta \mathrm{d} X \\
& +\int_{E}\left(\partial_{t} \mathcal{T}_{E}^{\alpha} \mathbf{u}\right) \cdot\left(\mathcal{T}_{E}^{\alpha} \mathbf{u}-\mathbf{u}\right) \zeta \mathrm{d} X
\end{aligned}
$$

where $\langle\cdot, \cdot\rangle$ denotes the usual duality pairing with respect to $G$.

\section{Remark 3.10.}

1. We have extended the functions $\mathbf{u}$ and $\mathbf{H}$ in the previous theorem by zero outside of $G \times(0, T)$.

2. If the open set $E \subset \mathbb{R}^{n+1}$ with $\mathcal{L}_{n+1}(E)<\infty$ satisfies for some $\Lambda>0$

$$
(G \times(0, T)) \cap \mathcal{O}_{\Lambda} \subset E \subset G \times(0, T),
$$

then all assertions of Theorem 3.9 remain valid with the exception of (3.16).

Before we prove Theorem 3.9 we need some preparatory results.

Lemma 3.11. Under the assumptions of Theorem 3.9 we have for all $Q_{i}^{\alpha}$ belonging to the Whitney covering of $E$ such that $Q_{i}^{\alpha} \cap K \neq \emptyset$

$$
f_{4 Q_{i}^{\alpha}}\left|\mathbf{u}-\mathbf{u}_{4 Q_{i}^{\alpha}}\right| \mathrm{d} X \leq c r_{i}^{\alpha}\left(\Lambda+\alpha^{-1} \delta_{\alpha, K}^{-n-3}\|\mathbf{u}\|_{L^{1}(E)}\right),
$$

where the constant $c$ depends only on $n$.

Proof. Let $Q_{i}^{\alpha} \subset E$ belonging to the Whitney covering be such that $Q_{i}^{\alpha} \cap K \neq \emptyset$. From property (W2) of the Whitney covering and (3.14) follows that (i) $16 Q_{i}^{\alpha} \cap$ $\left(\left(\mathcal{O}_{\Lambda}\right)^{c} \cap\left(\mathcal{U}_{1}\right)^{c}\right) \neq \emptyset$ or (ii) $16 Q_{i}^{\alpha} \cap(G \times(0, T))^{c} \neq \emptyset$, where the superscript ${ }^{c}$ 
denotes the complement in $\mathbb{R}^{n+1}$. In the case (i) we can use the Poincaré-type inequality (B.2) (cf. Appendix B) to estimate

$$
f_{4 Q_{i}^{\alpha}}\left|\mathbf{u}-\mathbf{u}_{4 Q_{i}^{\alpha}}\right| \mathrm{d} X \leq c r_{i}^{\alpha} f_{4 Q_{i}^{\alpha}}|\nabla \mathbf{u}|+\alpha|\mathbf{H}| \mathrm{d} X,
$$

where we used that $4 Q_{i}^{\alpha} \subset E \subset G \times(0, T)$. Since $16 Q_{i}^{\alpha} \cap\left(\left(\mathcal{O}_{\Lambda}\right)^{c} \cap\left(\mathcal{U}_{1}\right)^{c}\right) \neq \emptyset$ there exists $\hat{X} \in 16 Q_{i}^{\alpha} \cap\left(\mathcal{O}_{\Lambda}\right)^{c}$. Consequently, we have $4 Q_{i}^{\alpha} \subset Q_{20 r_{i}^{\alpha}}(\hat{X})$. From the above inequality and the definition of the maximal operator $\mathcal{M}^{*}$ and of the set $\mathcal{O}_{\Lambda}$ we obtain

$$
f_{4 Q_{i}^{\alpha}}\left|\mathbf{u}-\mathbf{u}_{4 Q_{i}^{\alpha}}\right| \mathrm{d} X \leq c r_{i}^{\alpha} f_{Q_{20 r_{i}^{\alpha}(\hat{X})}}|\nabla \mathbf{u}|+\alpha|\mathbf{H}| \mathrm{d} x \leq c r_{i}^{\alpha} \Lambda,
$$

which proves the assertion in the case (i). Let us now consider the case (ii). Since $Q_{i}^{\alpha} \cap K \neq \emptyset$ there exists $\hat{X} \in Q_{i}^{\alpha} \cap K$ and thus we derive

$$
\begin{aligned}
\delta_{\alpha, K} & \leq d_{\alpha}\left(\hat{X},(G \times(0, T))^{c}\right) \\
& \leq d_{\alpha}\left(\hat{X}, X_{i}^{\alpha}\right)+d_{\alpha}\left(X_{i}^{\alpha},(G \times(0, T))^{c}\right) \\
& \leq 17 r_{i}^{\alpha} .
\end{aligned}
$$

This, property (W2) of the Whitney covering, and $\mathcal{L}_{n+1}\left(4 Q_{i}^{\alpha}\right)=\omega_{n} \alpha\left(4 r_{i}^{\alpha}\right)^{n+2}$, where $\omega_{n}$ is the surface measure of the $(n-1)$-dimensional unit sphere, imply

$$
\begin{aligned}
f_{4 Q_{i}^{\alpha}}\left|\mathbf{u}-\mathbf{u}_{4 Q_{i}^{\alpha}}\right| \mathrm{d} X & \leq 2 f_{4 Q_{i}^{\alpha}}|\mathbf{u}| \mathrm{d} X \\
& \leq c \alpha^{-1}\left(r_{i}^{\alpha}\right)^{-n-3} r_{i}^{\alpha} \int_{E}|\mathbf{u}| \mathrm{d} X \\
& \leq c \alpha^{-1} \delta_{\alpha, K}^{-n-3} r_{i}^{\alpha}\|\mathbf{u}\|_{L^{1}(E)} .
\end{aligned}
$$

This proves the assertion in the case (ii), which finishes the proof.

Corollary 3.12. Under the assumptions of Theorem 3.9 we have for all $Q_{i}^{\alpha}$ with $Q_{i}^{\alpha} \cap K \neq \emptyset$ that

$$
f_{Q_{i}^{\alpha}}\left|\mathcal{T}_{E}^{\alpha} \mathbf{u}-\mathbf{u}\right| \mathrm{d} X \leq c r_{i}^{\alpha}\left(\Lambda+\alpha^{-1} \delta_{\alpha, K}^{-n-3}\|\mathbf{u}\|_{L^{1}(E)}\right)
$$

where the constant $c$ depends only on $n$.

Proof. This follows immediately from (3.11) and Lemma 3.11. 
Lemma 3.13. Let the assumptions of Theorem 3.9 be satisfied and let $Q_{i}^{\alpha}$ belonging to the Whitney covering of $E$ be such that $Q_{i}^{\alpha} \cap K \neq \emptyset$. Then we have for all $X \in Q_{i}^{\alpha} \cap K$

$$
\left|\mathcal{T}_{E}^{\alpha} \mathbf{u}(X)\right| \leq c\left(1+\alpha^{-1} \delta_{\alpha, K}^{-n-2}\|\mathbf{u}\|_{L^{1}(E)}\right),
$$

where the constant $c$ depends only on $n$.

Proof. We proceed as in the proof of Lemma 3.11. Let $Q_{i}^{\alpha} \cap K \neq \emptyset$. Again we have that (i) $16 Q_{i}^{\alpha} \cap\left(\left(\mathcal{O}_{\Lambda}\right)^{c} \cap\left(\mathcal{U}_{1}\right)^{c}\right) \neq \emptyset$ or (ii) $16 Q_{i}^{\alpha} \cap(G \times(0, T))^{c} \neq \emptyset$, where the superscript ${ }^{c}$ denotes the complement in $\mathbb{R}^{n+1}$. In the case (i) we use that since $16 Q_{i}^{\alpha} \cap\left(\left(\mathcal{O}_{\Lambda}\right)^{c} \cap\left(\mathcal{U}_{1}\right)^{c}\right) \neq \emptyset$ there exists $\hat{X} \in 16 Q_{i}^{\alpha} \cap\left(\mathcal{U}_{1}\right)^{c}$, and consequently we have $4 Q_{i}^{\alpha} \subset Q_{20 r_{i}^{\alpha}}^{\alpha}(\hat{X})$. From (3.6), the definition of the maximal operator $\mathcal{M}^{*}$ and of the set $\mathcal{U}_{1}$ we obtain

$$
\left|\mathcal{T}_{E}^{\alpha} \mathbf{u}(X)\right| \leq 8^{n+2} f_{4 Q_{i}^{\alpha}}|\mathbf{u}| \mathrm{d} X \leq 40^{n+2} f_{Q_{20 r_{i}^{\alpha}}^{\alpha}(\hat{X})}|\mathbf{u}| \mathrm{d} X \leq 40^{n+2}
$$

which proves the assertion in the case (i). In the second case we obtain as in (3.21)

$$
\begin{aligned}
\left|\mathcal{T}_{E}^{\alpha} \mathbf{u}(X)\right| & \leq c f_{4 Q_{i}^{\alpha}}|\mathbf{u}| \mathrm{d} X \\
& \leq c \alpha^{-1}\left(r_{i}^{\alpha}\right)^{-n-2} \int_{E}|\mathbf{u}| \mathrm{d} X \\
& \leq c \alpha^{-1} \delta_{\alpha, K}^{-n-2}\|\mathbf{u}\|_{L^{1}(E)},
\end{aligned}
$$

which proves the assertion in the case (ii). This finishes the proof.

For the next lemma we need a geometric property of an open set $U \subset \mathbb{R}^{n+1}$. One says that the set $U$ is of type $A$ with respect to the metric $d_{\alpha}$ (cf. [43]), if there exists a constant $A>0$ such that for all $0<r<\operatorname{diam}_{\alpha}(U)$ and all $X \in U$ there holds

$$
\mathcal{L}_{n+1}\left(Q_{r}^{\alpha}(X) \cap U\right) \geq A \mathcal{L}_{n+1}\left(Q_{r}^{\alpha}(X)\right)
$$

In the next lemma we show that the set $Q_{r_{0}}^{\alpha}\left(X_{0}\right)$ is of type A with respect to $d_{\alpha}$ with a constant $A=A(n)$.

Lemma 3.14. If for some $X \in \mathbb{R}^{n+1}, r>0$ and some $Q_{i}^{\alpha}$ belonging to the Whitney covering of $E$ holds $\frac{2}{3} Q_{i}^{\alpha} \cap Q_{r}^{\alpha}(X) \neq \emptyset$ and $Q_{r}^{\alpha}(X) \not \subset Q_{i}^{\alpha}$, then we have $r_{i}^{\alpha} \leq 6 r$ and there is a constant $c$ depending only on $n$ such that

$$
c \mathcal{L}_{n+1}\left(Q_{i}^{\alpha}\right) \leq \mathcal{L}_{n+1}\left(Q_{i}^{\alpha} \cap Q_{r}^{\alpha}(X)\right) \leq \mathcal{L}_{n+1}\left(Q_{i}^{\alpha}\right)
$$


Proof. From the the assumptions follows that there exist $\hat{X} \in \frac{2}{3} Q_{i}^{\alpha} \cap Q_{r}^{\alpha}(X)$ and $\hat{Y} \in Q_{r}^{\alpha}(X) \backslash Q_{i}^{\alpha}$. One easily sees that $Q_{\frac{1}{3} r_{i}^{\alpha}}^{\alpha}(\hat{X}) \subset Q_{i}^{\alpha}$, which implies

$$
\frac{r_{i}^{\alpha}}{3} \leq d_{\alpha}(\hat{X}, \hat{Y}) \leq d_{\alpha}(\hat{X}, X)+d_{\alpha}(X, \hat{Y}) \leq 2 r,
$$

which implies the first assertion. Moreover, we deduce from the fact that $Q_{r}^{\alpha}(X)$ is of type A with respect to $d_{\alpha}$ and $Q_{\frac{1}{3} r_{i}^{\alpha}}^{\alpha}(\hat{X}) \subset Q_{i}^{\alpha}=Q_{r_{i}^{\alpha}}^{\alpha}\left(X_{i}^{\alpha}\right)$ that

$$
\begin{aligned}
\mathcal{L}_{n+1}\left(Q_{i}^{\alpha} \cap Q_{r}^{\alpha}(X)\right) & \geq \mathcal{L}_{n+1}\left(Q_{\frac{1}{3} r_{i}^{\alpha}}^{\alpha}(\hat{X}) \cap Q_{r}^{\alpha}(X)\right) \\
& \geq c \mathcal{L}_{n+1}\left(Q_{\frac{1}{3} r_{i}^{\alpha}}^{\alpha}(\hat{X})\right) \\
& \geq c \mathcal{L}_{n+1}\left(Q_{i}^{\alpha}\right) .
\end{aligned}
$$

The upper bound is clear.

Now we are ready to prove Theorem 3.9.

\section{Proof of Theorem 3.9.}

Step 1 (Proof of (i)). We first prove assertion (i) for special compact sets $\overline{K_{0}^{\alpha}}$, where $K_{0}^{\alpha}:=Q_{r_{0}}^{\alpha}\left(X_{0}\right)$ with $r_{0}$ chosen such that $Q_{105 r_{0}}^{\alpha}\left(X_{0}\right) \subset G \times(0, T)$. Then we will cover a general compact set $K \subset G \times(0, T)$ by finitely many sets of that type and obtain the general assertion. Note that the set $K_{0}^{\alpha}$ is of type $A$ with respect to the metric $d_{\alpha}$ (cf. (3.24)). For open sets of type A the well-known theorem of Da Prato ( $c f$. [43, Theorem 3.1]) states that assertion (i) is equivalent to the statement that $\mathcal{T}_{E}^{\alpha} \mathbf{u} \in L_{\alpha}^{1,1+\frac{1}{n+2}}\left(K_{0}^{\alpha}\right)$. Thus we have to estimate $\mathcal{T}_{E}^{\alpha} \mathbf{u}$ in the norm of $L_{\alpha}^{1,1+\frac{1}{n+2}}\left(K_{0}^{\alpha}\right)$, which is given by $\|\cdot\|_{L_{\alpha}^{1,1+\frac{1}{n+2}}\left(K_{0}^{\alpha}\right)}:=\|\cdot\|_{L^{1}\left(K_{0}^{\alpha}\right)}+|\cdot|_{L_{\alpha}^{1,1+\frac{1}{n+2}}\left(K_{0}^{\alpha}\right)}$.

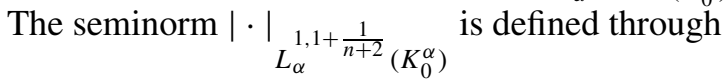

$$
|v|_{L_{\alpha}^{1,1+\frac{1}{n+2}}\left(K_{0}^{\alpha}\right)}:=\sup _{X \in K_{0}^{\alpha}, r>0} \mathcal{L}_{n+1}\left(K_{0}^{\alpha} \cap Q_{r}^{\alpha}(X)\right)^{-\left(1+\frac{1}{n+2}\right)} \int_{Q_{r}^{\alpha}(X) \cap K_{0}^{\alpha}} \mid v-v_{Q_{r}^{\alpha}(X) \cap K_{0}^{\alpha} \mid \mathrm{d} X .}
$$

Fix some $X \in K_{0}^{\alpha}=Q_{r_{0}}^{\alpha}\left(X_{0}\right)$. We have $K_{0}^{\alpha} \subset Q_{2 r_{0}}^{\alpha}(X)$. Thus we obtain for $r \geq 2 r_{0}$

$$
\begin{aligned}
& \mathcal{L}_{n+1}\left(K_{0}^{\alpha} \cap Q_{r}^{\alpha}(X)\right)^{-\frac{n+3}{n+2}} \int_{Q_{r}^{\alpha}(X) \cap K_{0}^{\alpha}}\left|\mathcal{T}_{E}^{\alpha} \mathbf{u}-\left(\mathcal{T}_{E}^{\alpha} \mathbf{u}\right)_{Q_{r}^{\alpha}(X) \cap K_{0}^{\alpha}}\right| \mathrm{d} y \\
& \leq 2 \mathcal{L}_{n+1}\left(K_{0}^{\alpha}\right)^{-\frac{n+3}{n+2}} \int_{K_{0}^{\alpha}}\left|\mathcal{T}_{E}^{\alpha} \mathbf{u}\right| \mathrm{d} X \leq c\left(K_{0}^{\alpha}, n\right)\|\mathbf{u}\|_{L^{1}\left(K_{0}^{\alpha}\right)},
\end{aligned}
$$


where we used a version of Lemma 3.5 for $p=1$ on the set $K_{0}^{\alpha}$. In order to estimate $\left|\mathcal{T}_{E}^{\alpha} \mathbf{u}\right|_{L_{\alpha}^{1, \frac{n+3}{n+2}}\left(K_{0}^{\alpha}\right)}$ it is thus sufficient to take the supremum over radii $0<r<2 r_{0}$. Let us abbreviate $Q_{r}^{\alpha}:=Q_{r}^{\alpha}(X)$ with $X \in K_{0}^{\alpha}$. Then there exists either (a) some $i \in \mathbb{N}$ such that $Q_{r}^{\alpha} \subset Q_{i}^{\alpha}$, with $Q_{i}^{\alpha}$ belonging to the Whitney covering of $E$ or (b) for all $i \in \mathbb{N}$ we have $Q_{r}^{\alpha} \not \subset Q_{i}^{\alpha}$, i.e. $Q_{r}^{\alpha} \backslash Q_{i}^{\alpha} \neq \emptyset$. Let us first consider the case (a). Since $K_{0}^{\alpha}$ is of type A with respect to $d_{\alpha}(c f$. (3.24)) we have

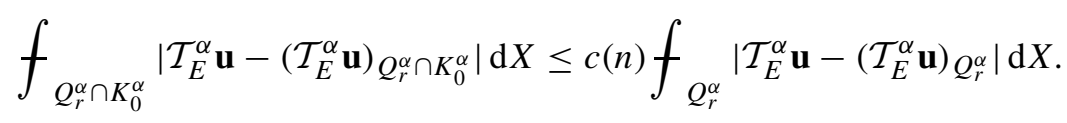

Using $Q_{r}^{\alpha} \subset Q_{i}^{\alpha}$, Lemma 3.6, and Lemma 3.11, which is possible since $K_{0}^{\alpha} \cap Q_{i}^{\alpha} \supset$ $K_{0}^{\alpha} \cap Q_{r}^{\alpha} \neq \emptyset$, we obtain

$$
\begin{aligned}
f_{Q_{r}^{\alpha}}\left|\mathcal{T}_{E}^{\alpha} \mathbf{u}-\left(\mathcal{T}_{E}^{\alpha} \mathbf{u}\right)_{Q_{r}^{\alpha}}\right| \mathrm{d} Y & \leq f_{Q_{r}^{\alpha}} f_{Q_{r}^{\alpha}}\left|\mathcal{T}_{E}^{\alpha} \mathbf{u}(Y)-\mathcal{T}_{E}^{\alpha} \mathbf{u}(Z)\right| \mathrm{d} Y \mathrm{~d} Z \\
& \leq c\left(r_{i}^{\alpha}\right)^{-1} r f_{4 Q_{i}^{\alpha}}\left|\mathbf{u}-\mathbf{u}_{4 Q_{i}^{\alpha}}\right| \mathrm{d} Y \\
& \leq c r\left(\Lambda+\alpha^{-1} \delta_{\alpha, K_{0}^{\alpha}}^{-n-3}\|\mathbf{u}\|_{L^{1}(E)}\right) \\
& =c\left(\alpha, n, K_{0}^{\alpha}, \Lambda,\|\mathbf{u}\|_{L^{1}(E)}\right) r
\end{aligned}
$$

Thus it remains to treat the second case (b). First observe that in this situation we have that

$$
Q_{34 r}^{\alpha} \cap((G \times(0, T)) \backslash E) \neq \emptyset .
$$

If this would not be the case, then we would have $Q_{34 r}^{\alpha}=Q_{34 r}^{\alpha}(X) \subset E$. Consequently there exists some $i \in \mathbb{N}$ such that $X \in \frac{1}{2} Q_{i}^{\alpha}$ due to property (W1) of the Whitney covering. Property (W2) and $Q_{34 r}^{\alpha} \subset E$ immediately imply that $34 r \leq 17 r_{i}^{\alpha}$, from which we deduce that for all $Y \in Q_{r}^{\alpha}$ holds

$$
d_{\alpha}\left(Y, X_{i}^{\alpha}\right) \leq d_{\alpha}(Y, X)+d_{\alpha}\left(X, X_{i}^{\alpha}\right)<r+\frac{r_{i}^{\alpha}}{2} \leq r_{i}^{\alpha}
$$

This means that $Q_{r}^{\alpha} \subset Q_{i}^{\alpha}$, which is a contradiction and (3.26) is proved.

We have

$$
\begin{aligned}
& f_{Q_{r}^{\alpha} \cap K_{0}^{\alpha}}\left|\mathcal{T}_{E}^{\alpha} \mathbf{u}-\left(\mathcal{T}_{E}^{\alpha} \mathbf{u}\right)_{Q_{r}^{\alpha} \cap K_{0}^{\alpha}}\right| \mathrm{d} Y \\
& \leq 2 f_{Q_{r}^{\alpha} \cap K_{0}^{\alpha}}\left|\mathcal{T}_{E}^{\alpha} \mathbf{u}-\mathbf{u}\right| \mathrm{d} Y+f_{Q_{r}^{\alpha} \cap K_{0}^{\alpha}} \mid \mathbf{u}-\mathbf{u}_{Q_{r}^{\alpha} \cap K_{0}^{\alpha} \mid \mathrm{d} Y} \\
& =: 2 I_{1}+I_{2} .
\end{aligned}
$$


Since $K_{0}^{\alpha}$ is of type A with respect to $d_{\alpha}$ we get

$$
I_{2} \leq c f_{Q_{r}^{\alpha}}\left|\mathbf{u}-\mathbf{u}_{Q_{r}^{\alpha}}\right| \mathrm{d} Y .
$$

From (3.26) follows the existence of a point $Z \in Q_{34 r}^{\alpha}(X) \cap((G \times(0, T)) \backslash E)$. Thus $Q_{r}^{\alpha}(X) \subset Q_{35 r}^{\alpha}(Z)$, which yields $Q_{35 r}^{\alpha}(Z) \subset Q_{105 r_{0}}^{\alpha}\left(X_{0}\right) \subset G \times(0, T)$, due to our assumptions on $r_{0}$. The above inequality and the Poincaré-type inequality (B.2) imply

$$
\begin{aligned}
I_{2} & \leq c f_{Q_{35 r}^{\alpha}(Z)}\left|\mathbf{u}-\mathbf{u}_{Q_{35 r}^{\alpha}(Z)}\right| \mathrm{d} Y \\
& \leq c r f_{35 Q_{r}^{\alpha}(Z)}|\nabla \mathbf{u}|+\alpha|\mathbf{H}| \mathrm{d} Y \\
& \leq \operatorname{cr} \Lambda,
\end{aligned}
$$

where we also used that $Z \in(G \times(0, T)) \backslash E \subset(G \times(0, T)) \backslash \mathcal{O}_{\Lambda}$. In order to estimate the term $I_{1}$ we use that the $\left\{\frac{2}{3} Q_{i}^{\alpha}\right\}$ cover $E$ and Lemma 3.14 to obtain

$$
\begin{aligned}
I_{1} & \leq c f_{Q_{r}^{\alpha}}\left|\mathcal{T}_{E}^{\alpha} \mathbf{u}(Y)-\mathbf{u}(Y)\right| \chi_{E} \chi_{K_{0}^{\alpha}} \mathrm{d} Y \\
& \leq c \sum_{i: \frac{2}{3} Q_{i}^{\alpha} \cap Q_{r}^{\alpha} \neq \emptyset} \frac{1}{\mathcal{L}_{n+1}\left(Q_{r}^{\alpha}\right)} \int_{Q_{r}^{\alpha} \cap \frac{2}{3} Q_{i}^{\alpha}}\left|\mathcal{T}_{E}^{\alpha} \mathbf{u}(Y)-\mathbf{u}(Y)\right| \chi_{K_{0}^{\alpha}} \mathrm{d} Y \\
& \leq c \sum_{i: \frac{2}{3} Q_{i}^{\alpha} \cap Q_{r}^{\alpha} \neq \emptyset} \frac{\mathcal{L}_{n+1}\left(Q_{r}^{\alpha} \cap Q_{i}^{\alpha}\right)}{\mathcal{L}_{n+1}\left(Q_{r}^{\alpha}\right)} f_{Q_{i}^{\alpha}}\left|\mathcal{T}_{E}^{\alpha} \mathbf{u}(Y)-\mathbf{u}(Y)\right| \chi_{K_{0}^{\alpha}} \mathrm{d} Y .
\end{aligned}
$$

For each $i \in \mathbb{N}$ with $\frac{2}{3} Q_{i}^{\alpha} \cap Q_{r}^{\alpha} \neq \emptyset$ we have using Corollary 3.12 together with Lemma 3.14

$$
\begin{aligned}
f_{Q_{i}^{\alpha}}\left|\mathcal{T}_{E}^{\alpha} \mathbf{u}(Y)-\mathbf{u}(Y)\right| \chi_{K_{0}^{\alpha}} \mathrm{d} Y & \leq c r_{i}^{\alpha}\left(\Lambda+\alpha^{-1} \delta_{\alpha, K_{0}^{\alpha}}^{-n-3}\|\mathbf{u}\|_{L^{1}(E)}\right) \\
& \leq 6 c r\left(\Lambda+\alpha^{-1} \delta_{\alpha, K_{0}^{\alpha}}^{-n-3}\|\mathbf{u}\|_{L^{1}(E)}\right) \\
& \leq c\left(\alpha, n, K_{0}^{\alpha}, \Lambda,\|\mathbf{u}\|_{L^{1}(E)}\right) r .
\end{aligned}
$$

Property (W4) of the Whitney decomposition implies that $\sum_{i} \chi_{Q_{i}^{\alpha}}(Y) \leq 120^{n+2}$. Thus we have

$$
\sum_{i: \frac{2}{3} Q_{i}^{\alpha} \cap Q_{r}^{\alpha} \neq \emptyset} \frac{\mathcal{L}_{n+1}\left(Q_{r}^{\alpha} \cap Q_{i}^{\alpha}\right)}{\mathcal{L}_{n+1}\left(Q_{r}^{\alpha}\right)}=\frac{1}{\mathcal{L}_{n+1}\left(Q_{r}^{\alpha}\right)} \int_{\mathbb{R}^{n+1}} \chi_{Q_{r}^{\alpha}} \sum_{i: \frac{2}{3} Q_{i}^{\alpha} \cap Q_{r}^{\alpha} \neq \emptyset} \chi_{Q_{i}^{\alpha}} \mathrm{d} Y \leq 120^{n+2}
$$


The last three estimates imply

$$
I_{1} \leq c\left(\alpha, n, K_{0}^{\alpha}, \Lambda,\|\mathbf{u}\|_{L^{1}(E)}\right) r
$$

which finishes the estimate of the seminorm $\left|\mathcal{T}_{E}^{\alpha} \mathbf{u}\right|_{L_{\alpha}{ }^{1,1+\frac{1}{n+2}}{ }_{\left(K_{0}^{\alpha}\right)}}$. Since $\mathcal{T}_{E}^{\alpha} \mathbf{u} \in$ $L^{1}\left(K_{0}^{\alpha}\right)$ due to Lemma 3.5, we proved $\mathcal{T}_{E}^{\alpha} \mathbf{u} \in C_{d_{\alpha}}^{0,1}\left(\overline{K_{0}^{\alpha}}\right)$.

In particular we have due to the definition of the metric $d_{\alpha}$ that for each $i=$ $1, \ldots, n$ and $|h| \leq h_{0}<r_{0}$ the difference quotient in spatial directions $h^{-1}\left(\mathcal{T}_{E}^{\alpha} \mathbf{u}(x+\right.$ $\left.\left.h e_{i}, t\right)-\mathcal{T}_{E}^{\alpha} \mathbf{u}(x, t)\right)$ is bounded in $L^{\infty}\left(K_{h_{0}}\right)$, where $K_{h_{0}}=\left\{X \in K_{0}^{\alpha} \mid \operatorname{dist}_{e}\left(X, \partial K_{0}^{\alpha}\right)>\right.$ $\left.h_{0}\right\}$ with dist $_{e}$ being the Euclidean distance in $\mathbb{R}^{n+1}$. This implies that $\nabla \mathcal{T}_{E}^{\alpha} \mathbf{u} \in$ $L^{\infty}\left(K_{0}^{\alpha}\right)$. That $\mathcal{T}_{E}^{\alpha} \mathbf{u}$ belongs to $L^{\infty}\left(K_{0}^{\alpha}\right)$ is obvious, since $K_{0}^{\alpha}$ is bounded and $\mathcal{T}_{E}^{\alpha} \mathbf{u} \in C_{d_{\alpha}}^{0,1}\left(K_{0}^{\alpha}\right)$.

Thus we proved assertion (i) for the special compact sets $\overline{K_{0}^{\alpha}}$.

In order to treat a general compact set $K \subset G \times(0, T)$ we cover $K$ by finitely many sets $K_{i}^{\alpha}:=\overline{Q_{s_{i}}^{\alpha}\left(Y_{i}\right)}, i=1, \ldots, N$ satisfying $Q_{210 s_{i}}^{\alpha}\left(Y_{i}\right) \subset G \times(0, T)$. Let $s_{0}:=\min \left\{s_{i} \mid i=1, \ldots, N\right\}$. If $d_{\alpha}(X, Y)<s_{0}$ and $X \in K_{i}^{\alpha}$ for some $i$, then we have $Y \in Q_{2 s_{i}}^{\alpha}\left(Y_{i}\right)$. Since for $Q_{2 s_{i}}^{\alpha}\left(Y_{i}\right)$ we have already proved assertion (i) we obtain

$$
\frac{\left|\mathcal{T}_{E}^{\alpha} \mathbf{u}(X)-\mathcal{T}_{E}^{\alpha} \mathbf{u}(Y)\right|}{d_{\alpha}(X, Y)} \leq c\left(K, \Lambda, \alpha, n,\|\mathbf{u}\|_{L^{1}(E)},\|\mathbf{u}\|_{L^{1}(\tilde{K} \times(0, T))}\right)<\infty .
$$

If $d_{\alpha}(X, Y) \geq s_{0}$ we have

$$
\left|\mathcal{T}_{E}^{\alpha} \mathbf{u}(X)-\mathcal{T}_{E}^{\alpha} \mathbf{u}(Y)\right| \leq \sup _{K} \mathcal{T}_{E}^{\alpha} \mathbf{u}-\inf _{K} \mathcal{T}_{E}^{\alpha} \mathbf{u} \leq c(K) \leq c\left(K, s_{0}\right) d_{\alpha}(X, Y),
$$

since $\mathcal{T}_{E}^{\alpha} \mathbf{u}$ is continuous on $K$. Thus we proved $\mathcal{T}_{E}^{\alpha} \mathbf{u} \in C_{d_{\alpha}}^{0,1}(K)$. That $\mathcal{T}_{E}^{\alpha} \mathbf{u}$ and $\nabla \mathcal{T}_{E}^{\alpha} \mathbf{u}$ belong to $L^{\infty}(K)$ follows in the same way as for the special sets $K_{0}^{\alpha}$.

This finishes the proof of assertion (i).

Step 2 (Proof of (3.15)). Since $\mathbf{u} \in L^{q}\left(0, T ; W^{1, q}(G)\right)$ and $\mathcal{T}_{E}^{\alpha} \mathbf{u}, \nabla \mathcal{T}_{E}^{\alpha} \mathbf{u} \in L^{\infty}(K)$ we obtain from an obvious modification of [37, Corollary 1.1.43] that almost everywhere on $K$ holds

$$
\begin{aligned}
\nabla \mathcal{T}_{E}^{\alpha} \mathbf{u} & =\chi_{E} \nabla \mathcal{T}_{E}^{\alpha} \mathbf{u}+\chi_{(G \times(0, T)) \backslash E} \nabla \mathcal{T}_{E}^{\alpha} \mathbf{u} \\
& =\chi_{E} \nabla \mathcal{T}_{E}^{\alpha} \mathbf{u}+\chi_{(G \times(0, T)) \backslash E} \nabla \mathbf{u} .
\end{aligned}
$$

From our assumption (3.14) on the set $E$ and the properties of the maximal operator $\mathcal{M}^{*}$ we get for a.e. $X \in K \cap((G \times(0, T)) \backslash E) \subset K \cap\left((G \times(0, T)) \backslash \mathcal{O}_{\Lambda}\right)$

$$
|\nabla \mathbf{u}(X)| \leq \mathcal{M}^{*}(|\nabla \mathbf{u}|)(X) \leq \Lambda \text {. }
$$

On the other hand for each $X \in K \cap E$ there exists some $i \in \mathbb{N}$, such that $X \in Q_{i}^{\alpha}$. Using an obvious modification of [37, Theorem 1.1.41] we get that the weak derivative and the classical derivative of $\mathcal{T}_{E}^{\alpha} \mathbf{u}$ in the $x$-directions coincide on $Q_{i}^{\alpha}$, and 
consequently we have, using the properties of the partition of unity $\psi_{j}$, properties (W6), (W3) and (W5) of the Whitney covering, $\left|\nabla \psi_{j}\right| \leq c\left(r_{j}^{\alpha}\right)^{-1}$, and Lemma 3.11 , that

$$
\begin{aligned}
\left|\nabla \mathcal{T}_{E}^{\alpha} \mathbf{u}(X)\right| & =\left|\nabla\left(\sum_{j \in A_{i}} \psi_{j}(X) \mathbf{u}_{Q_{j}^{\alpha}}\right)\right| \\
& =\left|\nabla\left(\sum_{j \in A_{i}} \psi_{j}(X)\left(\mathbf{u}_{Q_{j}^{\alpha}}-\mathbf{u}_{Q_{i}^{\alpha}}\right)\right)\right| \\
& =\left|\sum_{j \in A_{i}}\left(\nabla \psi_{j}\right)(X)\left(\mathbf{u}_{Q_{j}^{\alpha}}-\mathbf{u}_{Q_{i}^{\alpha}}\right)\right| \\
& \leq c(n) \sum_{j \in A_{i}}\left(r_{j}^{\alpha}\right)^{-1} f_{4 Q_{i}^{\alpha}}\left|\mathbf{u}(Y)-\mathbf{u}_{4 Q_{i}^{\alpha}}\right| \mathrm{d} Y \\
& \leq c(n)(120)^{n+2}\left(r_{i}^{\alpha}\right)^{-1} f_{4 Q_{i}^{\alpha}}\left|\mathbf{u}(Y)-\mathbf{u}_{4 Q_{i}^{\alpha}}\right| \mathrm{d} Y \\
& \leq c(n)\left(\Lambda+\alpha^{-1} \delta_{\alpha, K}^{-n-3} \| \mathbf{u}_{L^{1}(E)}\right),
\end{aligned}
$$

which together with (3.30) proves (3.15).

From our assumption on the set $E$ and the properties of the maximal operator $\mathcal{M}^{*}$ we get for a.e. $X \in K \cap((G \times(0, T)) \backslash E) \subset K \cap\left((G \times(0, T)) \backslash \mathcal{U}_{1}\right)$

$$
\left|\mathcal{T}_{E}^{\alpha} \mathbf{u}(X)\right|=|\mathbf{u}(X)| \leq \mathcal{M}^{*}(|\mathbf{u}|)(X) \leq 1 .
$$

On the other hand for each $X \in K \cap E$ there exists some $i \in \mathbb{N}$, such that $X \in Q_{i}^{\alpha}$. Lemma 3.13 yields for all $X \in K \cap Q_{i}^{\alpha}$

$$
\left|\mathcal{T}_{E}^{\alpha} \mathbf{u}(X)\right| \leq c\left(1+\alpha^{-1} \delta_{\alpha, K}^{-n-2}\|\mathbf{u}\|_{L^{1}(E)}\right) .
$$

The last two estimates prove (3.16).

Step 3 (Proof of (3.17)). Due to the definition of $\mathcal{T}_{E}^{\alpha} \mathbf{u}$ we have $\mathcal{T}_{E}^{\alpha} \mathbf{u}=\mathbf{u}$ on $(G \times(0, T)) \backslash E$ and that the Lipschitz truncation $\mathcal{T}_{E}^{\alpha} \mathbf{u}$ is smooth in $E$. In particular the classical derivative $\partial_{t} \mathcal{T}_{E}^{\alpha} \mathbf{u}$ exists in $E$. Using the definition of $\mathcal{T}_{E} \mathbf{u}$, Lemma 3.8, estimate (3.11), Lemma 3.11, and property (W5) of the Whitney covering one 
estimates

$$
\begin{aligned}
& \int_{K \cap E}\left|\partial_{t} \mathcal{T}_{E}^{\alpha} \mathbf{u}(X) \cdot\left(\mathcal{T}_{E}^{\alpha} \mathbf{u}(X)-\mathbf{u}(X)\right)\right| \mathrm{d} X \\
& \leq \sum_{j: Q_{j}^{\alpha} \cap K \neq \emptyset} \int_{Q_{j}^{\alpha}}\left|\partial_{t} \mathcal{T}_{E}^{\alpha} \mathbf{u}(X)\right|\left|\mathcal{T}_{E}^{\alpha} \mathbf{u}(X)-\mathbf{u}(X)\right| \mathrm{d} X \\
& \leq c, \alpha^{-1} \sum_{j: Q_{j}^{\alpha} \cap K \neq \emptyset}\left(r_{j}^{\alpha}\right)^{-2} f_{4 Q_{j}^{\alpha}}\left|\mathbf{u}-\mathbf{u}_{4 Q_{j}^{\alpha}}\right| \mathrm{d} Y \int_{Q_{j}^{\alpha}}\left|\mathcal{T}_{E}^{\alpha} \mathbf{u}(X)-\mathbf{u}(X)\right| \mathrm{d} X \\
& \leq c \alpha^{-1} \sum_{j: Q_{j}^{\alpha} \cap K \neq \emptyset}\left(r_{j}^{\alpha}\right)^{-2} \mathcal{L}_{n+1}\left(Q_{j}^{\alpha}\right)\left(f_{4 Q_{j}^{\alpha}}\left|\mathbf{u}-\mathbf{u}_{4 Q_{j}^{\alpha}}\right| \mathrm{d} Y\right)^{2} \\
& \leq c \alpha^{-1} \sum_{j: Q_{j}^{\alpha} \cap K \neq \emptyset} \mathcal{L}_{n+1}\left(Q_{j}^{\alpha}\right)\left(\Lambda+\alpha^{-1} \delta_{\alpha, K}^{-n-3}\|\mathbf{u}\|_{L^{1}(E)}\right)^{2} \\
& \leq c \alpha^{-1} \mathcal{L}_{n+1}(E)\left(\Lambda+\alpha^{-1} \delta_{\alpha, K}^{-n-3}\|\mathbf{u}\|_{L^{1}(E)}\right)^{2} .
\end{aligned}
$$

This proves the estimate (3.17).

Step 4 (Proof of (3.18)). Let $0<h<T$. Given $f \in L^{1}(G \times(0, T))$ the Steklov average $f_{h}$ of $f$ is defined by

$$
f_{h}(x, t):=\frac{1}{h} \int_{t}^{t+h} f(x, s) \mathrm{d} s, \quad(x, t) \in G \times(0, T)
$$

where $f$ has been extended by zero outside of $(0, T)$. Then we have

$$
\partial_{t} f_{h}(x, t)=h^{-1}(f(x, t+h)-f(x, t)) .
$$

From the assumptions on $\mathbf{H}$ and $\mathbf{u}$ follows that $\mathbf{H}_{h} \in W^{1, \sigma}\left(0, T ; L^{\sigma}(G)\right)$ and $\mathbf{u}_{h} \in$ $W^{1, \infty}\left(0, T ; L^{2}(G)\right) \cap W^{1, q}\left(0, T ; W^{1, q}(G)\right)$. Let $\zeta \in C_{0}^{\infty}(G \times(0, T))$ be fixed. Then there exists a compact set $K$ such that $\operatorname{supp}(\zeta) \subset \subset$ int $K \subset \subset G \times(0, T)$. Set

$$
d_{0}^{\alpha}:=d_{\alpha}(\operatorname{supp}(\zeta), \partial K)
$$

Let $0<h<\frac{1}{2} \min \left\{d_{0}^{\alpha}, \alpha\left(d_{0}^{\alpha}\right)^{2}\right\}$. From (3.13) follows by density arguments that the weak derivative $\partial_{t} \mathbf{u}$ belongs to $L^{\sigma}\left(0, T ;\left(W_{0}^{1, \sigma^{\prime}}(G)\right)^{*}\right)$. From assertion (i) of Theorem 3.9 follows that $\left(\left(\mathcal{T}_{E}^{\alpha} \mathbf{u}\right)_{h} \zeta\right)_{-h}$ is an admissible test function for $\partial_{t} \mathbf{u}$. Using the properties of the Steklov average, Lemma 3.5, and $\mathcal{T}_{E}^{\alpha} \mathbf{u} \in L^{\infty}(K)$ one 
easily justifies the following calculations

$$
\begin{aligned}
\int_{0}^{T} & \left\langle\partial_{t} \mathbf{u}(t),\left(\left(\mathcal{T}_{E}^{\alpha} \mathbf{u}\right)_{h}(t) \zeta(t)\right)_{-h}\right\rangle \mathrm{d} t \\
= & \int_{0}^{T}\left\langle\partial_{t} \mathbf{u}_{h}(t),\left(\mathcal{T}_{E}^{\alpha} \mathbf{u}\right)_{h}(t) \zeta(t)\right\rangle \mathrm{d} t \\
= & \int_{G \times(0, T)} \partial_{t} \mathbf{u}_{h} \cdot \mathbf{u}_{h} \zeta \mathrm{d} X+\int_{G \times(0, T)} \partial_{t} \mathbf{u}_{h} \cdot\left(\mathcal{T}_{E}^{\alpha} \mathbf{u}-\mathbf{u}\right)_{h} \zeta \mathrm{d} X \\
= & \frac{1}{2} \int_{G \times(0, T)} \partial_{t}\left|\mathbf{u}_{h}\right|^{2} \zeta \mathrm{d} X-\frac{1}{2} \int_{G \times(0, T)} \partial_{t}\left|\left(\mathcal{T}_{E}^{\alpha} \mathbf{u}-\mathbf{u}\right)_{h}\right|^{2} \zeta \mathrm{d} X \\
& +\int_{G \times(0, T)} \partial_{t}\left(\mathcal{T}_{E}^{\alpha} \mathbf{u}\right)_{h} \cdot\left(\mathcal{T}_{E}^{\alpha} \mathbf{u}-\mathbf{u}\right)_{h} \zeta \mathrm{d} X \\
= & -\frac{1}{2} \int_{G \times(0, T)}\left(\left|\mathbf{u}_{h}\right|^{2}-\left|\left(\mathcal{T}_{E}^{\alpha} \mathbf{u}-\mathbf{u}\right)_{h}\right|^{2}\right) \partial_{t} \zeta \mathrm{d} X \\
& +\int_{E}\left(\partial_{t}\left(\mathcal{T}_{E}^{\alpha} \mathbf{u}\right)_{h} \zeta\right)_{-h} \cdot\left(\mathcal{T}_{E}^{\alpha} \mathbf{u}-\mathbf{u}\right)^{\mathrm{d} X},
\end{aligned}
$$

where we used as the last step that $\mathcal{T}_{E}^{\alpha} \mathbf{u}=\mathbf{u}$ in $(G \times(0, T)) \backslash E$. Next, by the well-known properties of the Steklov average one finds for $h \rightarrow 0$

$$
\begin{array}{rlrl}
\partial_{t} \mathbf{u}_{h} & \rightarrow \partial_{t} \mathbf{u} & \text { in } & L^{\sigma}\left(0, T ;\left(W_{0}^{1, \sigma^{\prime}}(G)\right)^{*}\right), \\
\left(\mathcal{T}_{E}^{\alpha} \mathbf{u}\right)_{h} \zeta & \rightarrow\left(\mathcal{T}_{E}^{\alpha} \mathbf{u}\right) \zeta & \text { in } \quad L^{\sigma^{\prime}}\left(0, T ; W_{0}^{1, \sigma^{\prime}}(G)\right), \\
\mathbf{u}_{h} \rightarrow \mathbf{u} & \text { in } \quad L^{2}(K), \\
\left(\mathcal{T}_{E}^{\alpha} \mathbf{u}\right)_{h} \rightarrow\left(\mathcal{T}_{E}^{\alpha} \mathbf{u}\right) & \text { in } \quad L^{2}(K) .
\end{array}
$$

Therefore,

$$
\begin{aligned}
\int_{0}^{T}\left\langle\partial_{t} \mathbf{u}(t),\left(\mathcal{T}_{E}^{\alpha} \mathbf{u}\right)(t) \zeta(t)\right\rangle \mathrm{d} t= & -\frac{1}{2} \int_{G \times(0, T)}\left(|\mathbf{u}|^{2}-\left|\mathcal{T}_{E}^{\alpha} \mathbf{u}-\mathbf{u}\right|^{2} \partial_{t} \zeta \mathrm{d} X\right. \\
& +\lim _{h \rightarrow 0} \int_{E}\left(\partial_{t}\left(\mathcal{T}_{E}^{\alpha} \mathbf{u}\right)_{h} \zeta\right)_{-h} \cdot\left(\mathcal{T}_{E}^{\alpha} \mathbf{u}-\mathbf{u}\right) \mathrm{d} X
\end{aligned}
$$

Thus, it only remains to verify that

$$
\lim _{h \rightarrow 0} \int_{E}\left(\partial_{t}\left(\mathcal{T}_{E}^{\alpha} \mathbf{u}\right)_{h} \zeta\right)_{-h} \cdot\left(\mathcal{T}_{E}^{\alpha} \mathbf{u}-\mathbf{u}\right) \mathrm{d} X=\int_{E} \partial_{t} \mathcal{T}_{E}^{\alpha} \mathbf{u} \cdot\left(\mathcal{T}_{E}^{\alpha} \mathbf{u}-\mathbf{u}\right) \zeta \mathrm{d} X
$$

We define for almost all $(x, t) \in E$

$$
\begin{aligned}
\boldsymbol{\theta}_{h}^{\alpha}(x, t): & =\left(\partial_{t}\left(\mathcal{T}_{E}^{\alpha} \mathbf{u}\right)_{h} \zeta\right)_{-h}(x, t) \\
& =\int_{0}^{1} \frac{\left(\mathcal{T}_{E}^{\alpha} \mathbf{u}\right)(x, t+(1-\tau) h)-\left(\mathcal{T}_{E}^{\alpha} \mathbf{u}\right)(x, t-\tau h)}{h} \zeta(x, t-\tau h) \mathrm{d} \tau
\end{aligned}
$$


and set

$$
A^{\alpha}:=\left\{(x, t+s) \mid(x, t) \in \operatorname{supp}(\zeta), 0 \leq s \leq \alpha \frac{\left(d_{0}^{\alpha}\right)^{2}}{2}\right\}
$$

Since $h<\alpha \frac{\left(d_{0}^{\alpha}\right)^{2}}{2}$ we have $\operatorname{supp}\left(\boldsymbol{\theta}_{h}^{\alpha}\right) \subset A^{\alpha} \subset K \subset G \times(0, T)$. Using these notations and the property of the partition of unity $\left\{\psi_{i}\right\}$ one gets

$$
\int_{E}\left(\partial_{t}\left(\mathcal{T}_{E}^{\alpha} \mathbf{u}\right)_{h} \zeta\right)_{-h} \cdot\left(\mathcal{T}_{E}^{\alpha} \mathbf{u}-\mathbf{u}\right) \mathrm{d} X=\sum_{i} \int_{E} \psi_{i} \boldsymbol{\theta}_{h}^{\alpha} \cdot\left(\mathcal{T}_{E}^{\alpha} \mathbf{u}-\mathbf{u}\right) \mathrm{d} X .
$$

We split the sum on the right-hand side of this equation into two parts by defining the following set of indices

$$
\mathcal{S}_{h}^{\alpha}:=\left\{i \in \mathbb{N} \mid r_{i}^{\alpha} \leq 2 h^{1 / 2}, Q_{i}^{\alpha} \cap K \neq \emptyset\right\}
$$

With this notation we have

$$
\begin{aligned}
\int_{E}\left(\partial_{t}\left(\mathcal{T}_{E}^{\alpha} \mathbf{u}\right)_{h} \zeta\right)_{-h} \cdot\left(\mathcal{T}_{E}^{\alpha} \mathbf{u}-\mathbf{u}\right) \mathrm{d} X & =\sum_{i \in \mathcal{S}_{h}^{\alpha}} \int_{E} \psi_{i} \boldsymbol{\theta}_{h}^{\alpha} \cdot\left(\mathcal{T}_{E}^{\alpha} \mathbf{u}-\mathbf{u}\right) \mathrm{d} X \\
& +\sum_{i \in\left(\mathcal{S}_{h}^{\alpha}\right)^{c}} \int_{E} \psi_{i} \boldsymbol{\theta}_{h}^{\alpha} \cdot\left(\mathcal{T}_{E}^{\alpha} \mathbf{u}-\mathbf{u}\right) \mathrm{d} X \\
& =I_{h}^{\alpha}+I I_{h}^{\alpha} .
\end{aligned}
$$

Due to the properties of $\zeta,(3.34)$, and $\mathcal{T}_{E}^{\alpha} \mathbf{u} \in \operatorname{Lip}_{d_{\alpha}}(K)$ we get a.e. in $E$

$$
\left|\boldsymbol{\theta}_{h}^{\alpha}\right| \leq c h^{-\frac{1}{2}} .
$$

For $i \in \mathcal{S}_{h}^{\alpha}$ we have due to (3.22) and $r_{i}^{\alpha} \leq 2 h^{\frac{1}{2}}$

$$
\begin{aligned}
\int_{Q_{i}^{\alpha} \cap K} \psi_{i}\left|\mathcal{T}_{E}^{\alpha} \mathbf{u}-\mathbf{u}\right| \mathrm{d} X & \leq c r_{i}^{\alpha} \mathcal{L}_{n+1}\left(Q_{i}^{\alpha}\right)\left(\Lambda+\alpha^{-1} \delta_{\alpha, K}^{-n-3}\|\mathbf{u}\|_{L^{1}(E)}\right) \\
& \leq \operatorname{ch}^{\frac{1}{2}} \mathcal{L}_{n+1}\left(Q_{i}^{\alpha}\right)\left(\Lambda+\alpha^{-1} \delta_{\alpha, K}^{-n-3}\|\mathbf{u}\|_{L^{1}(E)}\right)
\end{aligned}
$$

The last two estimates yield

$$
\left|I_{h}^{\alpha}\right| \leq c \sum_{i \in \mathcal{S}_{h}^{\alpha}} \mathcal{L}_{n+1}\left(Q_{i}^{\alpha}\right) \leq c \mathcal{L}_{n+1}(E)<\infty
$$

where we also used property (W5) of the Whitney decomposition and $\mathcal{L}_{n+1}(E)<$ $\infty$. Since $\mathcal{S}_{h}^{\alpha} \rightarrow \emptyset$ for $h \rightarrow 0$ it follows

$$
\lim _{h \rightarrow 0^{+}} I_{h}^{\alpha}=0
$$


Since $\mathcal{T}_{E}^{\alpha} \mathbf{u}$ is smooth on $E$ one immediately finds from (3.34) and the definition of $\mathcal{S}_{h}^{\alpha}$, that

$$
\sum_{i \in\left(\mathcal{S}_{h}^{\alpha}\right)^{c}} \psi_{i} \boldsymbol{\theta}_{h}^{\alpha} \cdot\left(\mathcal{T}_{E}^{\alpha} \mathbf{u}-\mathbf{u}\right) \rightarrow \partial_{t} \mathcal{T}_{E}^{\alpha} \mathbf{u} \cdot\left(\mathcal{T}_{E}^{\alpha} \mathbf{u}-\mathbf{u}\right) \zeta \quad \text { a. e. in } E \quad \text { as } \quad h \rightarrow 0 .
$$

For $i \in\left(\mathcal{S}_{h}^{\alpha}\right)^{c}$ we get due to (3.34) and $0<h<\alpha \frac{\left(r_{i}^{\alpha}\right)^{2}}{2}$ that

$$
\left|\psi_{i} \theta_{h}^{\alpha}\right| \leq \max _{Y \in Q_{i}^{\alpha} \cap K}\left|\left(\partial_{t} \mathcal{T}_{E}^{\alpha} \mathbf{u}\right)(Y)\right|,
$$

from which follows by (3.12) together with (3.19)

$$
\left|\psi_{i} \boldsymbol{\theta}_{h}\right| \leq c\left(r_{i}^{\alpha}\right)^{-1} \chi Q_{i}^{\alpha} \chi_{K} .
$$

Thus, by the properties of the Whitney covering and Corollary 3.12 we get

$$
\begin{aligned}
\int_{K}\left|\sum_{i \in\left(\mathcal{S}_{h}^{\alpha}\right)^{c}} \psi_{i} \boldsymbol{\theta}_{h} \cdot\left(\mathcal{T}_{E}^{\alpha} \mathbf{u}-\mathbf{u}\right)\right| \mathrm{d} X & \leq c \int_{K} \sum_{i}\left(r_{i}^{\alpha}\right)^{-1}\left|\mathcal{T}_{E}^{\alpha} \mathbf{u}-\mathbf{u}\right| \chi_{Q_{i}^{\alpha}} \mathrm{d} X \\
& \leq c \sum_{i} \mathcal{L}_{n+1}\left(Q_{i}^{\alpha}\right) \\
& \leq c \mathcal{L}_{n+1}(E)<\infty .
\end{aligned}
$$

Now Lebesgue's theorem of dominated convergence implies

$$
\lim _{h \rightarrow 0^{+}} I I_{h}^{\alpha}=\int_{E} \partial_{t} \mathcal{T}_{E}^{\alpha} \mathbf{u} \cdot\left(\mathcal{T}_{E}^{\alpha} \mathbf{u}-\mathbf{u}\right) \zeta \mathrm{d} X .
$$

This completes the proof of Theorem 3.9.

\section{Proof of the main theorem}

Since for $q>\frac{2(n+1)}{n+2}$ the proof of Theorem 1.3 has already been carried out in [52] it will be enough to prove the theorem for the case $q<2$. Throughout this section let $\frac{2 n}{n+2}<q<2$ be fixed. Recall $Q_{T}=\Omega \times(0, T)$.

Let $\Phi \in C^{\infty}([0, \infty))$ be a non-increasing function, such that $0 \leq \Phi \leq 1$ in $[0, \infty), \Phi \equiv 1$ on $[0,1], \Phi \equiv 0$ in $[2, \infty)$ and $0 \leq-\Phi^{\prime} \leq 2$. For $m \in \mathbb{N}$ we set

$$
\Phi_{m}(\tau):=\Phi(\tau / m), \quad \tau \in[0, \infty) .
$$

Let $\mathbf{u}_{0} \in L^{2}(\Omega)$ and let $\mathbf{F} \in L^{q^{\prime}}\left(Q_{T}\right)$. In [52, Theorem 3.1] $]^{6}$, it has been proved that there exists a unique weak solution $\mathbf{u}_{m} \in L^{q}\left(0, T ; V_{q}\right) \cap C([0, T] ; H)$ to the

${ }^{6}$ Since $\Omega$ is a bounded open set, one easily verifies that the assumptions (I)-(IV) on $\mathbf{S}$ here imply the assumptions (I)-(IV) on $\mathbf{S}$ in [52]. 
system

$$
\begin{array}{rlrl}
\operatorname{div} \mathbf{u}_{m} & =0 & & \text { in } Q_{T}, \\
\partial_{t} \mathbf{u}_{m}+\operatorname{div}\left(\mathbf{u}_{m} \otimes \mathbf{u}_{m} \Phi_{m}\left(\left|\mathbf{u}_{m}\right|\right)-\mathbf{S}\left(\mathbf{D}\left(\mathbf{u}_{m}\right)\right)+\mathbf{F}\right) & =-\nabla p_{m} & & \text { in } Q_{T}, \\
\left.\mathbf{u}_{m}\right|_{\partial \Omega \times(0, T)} & =0, & \\
\mathbf{u}_{m}(0) & =\mathbf{u}_{0} & & \text { in } \Omega,
\end{array}
$$

i.e. the following identity

$$
\begin{aligned}
& -\int_{Q_{T}} \mathbf{u}_{m} \cdot \partial_{t} \boldsymbol{\varphi} \mathrm{d} x \mathrm{~d} t+\int_{Q_{T}}\left(\mathbf{S}\left(\mathbf{D}\left(\mathbf{u}_{m}\right)\right)-\mathbf{u}_{m} \otimes \mathbf{u}_{m} \Phi_{m}\left(\left|\mathbf{u}_{m}\right|\right)\right): \mathbf{D}(\boldsymbol{\varphi}) \mathrm{d} x \mathrm{~d} t \\
& =\int_{Q_{T}} \mathbf{F}: \nabla \boldsymbol{\varphi} \mathrm{d} x \mathrm{~d} t+\int_{\Omega} \mathbf{u}_{0} \cdot \boldsymbol{\varphi}(0) \mathrm{d} x
\end{aligned}
$$

holds for every $\varphi \in C^{\infty}\left(Q_{T}\right)$ with $\operatorname{div} \varphi=0$ and $\operatorname{supp}(\varphi) \subset \subset \Omega \times[0, T)$. Furthermore, it has been proved there that for all $0<t<T$

$$
\begin{aligned}
& \frac{1}{2}\left\|\mathbf{u}_{m}(t)\right\|_{H}^{2}+\int_{0}^{t} \int_{\Omega} \mathbf{S}\left(\mathbf{D}\left(\mathbf{u}_{m}\right)\right): \mathbf{D}\left(\mathbf{u}_{m}\right) \mathrm{d} x \mathrm{~d} s \\
& =\frac{1}{2}\left\|\mathbf{u}_{0}\right\|_{H}^{2}+\int_{0}^{t} \int_{\Omega} \mathbf{F}: \nabla \mathbf{u}_{m} \mathrm{~d} x \mathrm{~d} s .
\end{aligned}
$$

In what follows let $c$ denote a positive constant, which may vary from line to line but does not depend on the parameter $m \in \mathbb{N}$. Observing the coercivity condition (III) from (4.6) we deduce

$$
\left\|\mathbf{u}_{m}\right\|_{L^{\infty}(0, T ; H)}^{2}+\left\|\mathbf{D}\left(\mathbf{u}_{m}\right)\right\|_{L^{q}\left(Q_{T}\right)}^{q} \leq c .
$$

By virtue Korn's inequality and Sobolev's embedding theorem we get

$$
\left\|\mathbf{u}_{m}\right\|_{L^{q}\left(0, T ; W^{1, q}(\Omega)\right)}+\left\|\mathbf{u}_{m}\right\|_{L^{q}\left(0, T ; L^{q^{*}}(\Omega)\right)} \leq c,
$$

where $q^{*}=\frac{n q}{n-q}$ is the embedding exponent. With help of Sobolev's inequalities along with the Hölder's inequality taking into account (4.7) and (4.8) we estimate

$$
\left\|\mathbf{u}_{m}\right\|_{L^{q \frac{n+2}{n}}\left(Q_{T}\right)} \leq c .
$$

From the growth condition (II) of $\mathbf{S}$ and (4.7) follows

$$
\left\|\mathbf{S}\left(\mathbf{D}\left(\mathbf{u}_{m}\right)\right)\right\|_{L^{q^{\prime}}\left(Q_{T}\right)} \leq c .
$$

By means of reflexivity we can pass to a subsequence (which we denote for simplicity of notation still by $\mathbf{u}_{m}$ and $\left.\mathbf{S}_{m}\right)$ and functions $\mathbf{u} \in L^{q}\left(0, T ; V_{q}\right) \cap L^{\infty}(0, T ; H)$, 
$\tilde{\mathbf{S}} \in L^{q^{\prime}}\left(Q_{T}\right)$ and $\tilde{\mathbf{H}} \in L^{q \frac{n+2}{2 n}}\left(Q_{T}\right)$ such that for $m \rightarrow \infty$

$$
\begin{aligned}
\mathbf{D}\left(\mathbf{u}_{m}\right) & \rightarrow \mathbf{D}(\mathbf{u}) & \text { weakly in } L^{q}\left(Q_{T}\right), \\
\mathbf{u}_{m} & \rightarrow \mathbf{u} & \text { weakly in } L^{q \frac{n+2}{n}}\left(Q_{T}\right), \\
\mathbf{S}\left(\cdot, \mathbf{D}\left(\mathbf{u}_{m}\right)\right) & \rightarrow \tilde{\mathbf{S}} & \text { weakly in } L^{q^{\prime}}\left(Q_{T}\right), \\
\mathbf{u}_{m} \otimes \mathbf{u}_{m} \Phi_{m}\left(\left|\mathbf{u}_{m}\right|\right) & \rightarrow \tilde{\mathbf{H}} & \text { weakly in } L^{q \frac{n+2}{2 n}}\left(Q_{T}\right) .
\end{aligned}
$$

Passing to the limit $m \rightarrow \infty$ in (4.5) thus gives

$$
\begin{aligned}
& -\int_{Q_{T}} \mathbf{u} \cdot \partial_{t} \boldsymbol{\phi} \mathrm{d} x \mathrm{~d} t+\int_{Q_{T}}(\tilde{\mathbf{S}}-\tilde{\mathbf{H}}): \mathbf{D}(\boldsymbol{\phi}) \mathrm{d} x \mathrm{~d} t \\
& =\int_{Q_{T}} \mathbf{F}: \nabla \boldsymbol{\phi} \mathrm{d} x \mathrm{~d} t+\int_{\Omega} \mathbf{u}_{0} \cdot \boldsymbol{\phi}(0) \mathrm{d} x
\end{aligned}
$$

for all $\phi \in C^{\infty}\left(Q_{T}\right)$ with $\operatorname{div} \phi=0$ and $\operatorname{supp}(\phi) \subset \subset \Omega \times[0, T)$. From this identity one can show that $\mathbf{u} \in C_{w}([0, T] ; H)(c f$. [52, Section 4]). In particular we have $\mathbf{u}(0)=\mathbf{u}_{0}$.

Next, let $G \subset \subset \Omega$ be a fixed but arbitrary open bounded set. Clearly we may assume there exists an open bounded set $G^{\prime} \subset \subset \Omega$ with $G \subset \subset G^{\prime}$ and $\partial G^{\prime} \in$ $C^{2}$. Since $q>\frac{2 n}{n+2}$ we can find $\sigma_{0}>1$ with $q \leq 2 \sigma_{0}<q \frac{n+2}{n}$. Using (4.9), (4.10), $q<2$, and the properties of $\sigma_{0}$ we get from (4.5) that the weak derivative $\partial_{t} \mathbf{u}_{m}$ belong to $L^{\sigma_{0}}\left(0, T ;\left(V_{\sigma_{0}^{\prime}}\left(G^{\prime}\right)\right)^{*}\right)$. This, the compact embedding $V_{q}\left(G^{\prime}\right) \hookrightarrow \hookrightarrow$ $H_{r}\left(G^{\prime}\right), 1 \leq r<q^{*}$, the Aubin-Lions compactness lemma (cf. [30]) and a parabolic interpolation result using (4.10), yield $\tilde{\mathbf{H}}=\mathbf{u} \otimes \mathbf{u}$ and for $m \rightarrow \infty$ (cf. [52, Section 4] for a different reasoning)

$$
\begin{array}{rlrl}
\mathbf{u}_{m} & \rightarrow \mathbf{u} & & \text { strongly in } L^{2 \sigma_{0}}\left(0, T ; L^{2 \sigma_{0}}\left(G^{\prime}\right)\right), \\
\mathbf{u}_{m} \otimes \mathbf{u}_{m} \Phi_{m}\left(\left|\mathbf{u}_{m}\right|\right) & \rightarrow \mathbf{u} \otimes \mathbf{u} & \text { strongly in } L^{\sigma_{0}}\left(0, T ; L^{\sigma_{0}}\left(G^{\prime}\right)\right) .
\end{array}
$$

From (4.13) and (4.7) follows again by parabolic interpolation

$$
\mathbf{u}_{m} \rightarrow \mathbf{u} \quad \text { strongly in } L^{r}\left(0, T ; L^{2}\left(G^{\prime}\right)\right), \quad 1 \leq r<\infty
$$

Moreover, combining (4.5) and (4.12) yields

$$
\begin{aligned}
& -\int_{Q_{T}}\left(\mathbf{u}_{m}-\mathbf{u}\right) \cdot \partial_{t} \boldsymbol{\varphi} \mathrm{d} x \mathrm{~d} t+\int_{Q_{T}}\left(\mathbf{S}\left(\mathbf{D}\left(\mathbf{u}_{m}\right)\right)-\tilde{\mathbf{S}}\right): \nabla \boldsymbol{\varphi} \mathrm{d} x \mathrm{~d} t \\
& =\int_{Q_{T}}\left(\mathbf{u}_{m} \otimes \mathbf{u}_{m} \Phi_{m}\left(\left|\mathbf{u}_{m}\right|\right)-\mathbf{u} \otimes \mathbf{u}\right): \nabla \boldsymbol{\varphi} \mathrm{d} x \mathrm{~d} t
\end{aligned}
$$


for all $\varphi \in C^{\infty}\left(Q_{T}\right)$ with $\operatorname{div} \varphi=0$ and $\operatorname{supp}(\varphi) \subset \subset \Omega \times[0, T)$. From Theorem 2.2 in Section 2 one gets unique functions

$$
\begin{aligned}
& p_{1, m} \in L^{q^{\prime}}\left(0, T ; L^{q^{\prime}}\left(G^{\prime}\right)\right), \\
& p_{2, m} \in L^{\sigma_{0}}\left(0, T ; L^{\sigma_{0}}\left(G^{\prime}\right)\right), \\
& p_{h, m} \in C_{w}\left([0, T] ; W^{1,2}\left(G^{\prime}\right)\right)
\end{aligned}
$$

with $\Delta p_{h, m}=0$ and $p_{h, m}(0)=0$ and

$$
\begin{aligned}
& -\int_{0}^{T} \int_{G^{\prime}}\left(\mathbf{u}_{m}-\mathbf{u}\right) \cdot \partial_{t} \boldsymbol{\phi} \mathrm{d} x \mathrm{~d} t+\int_{0}^{T} \int_{G^{\prime}}\left(\mathbf{S}\left(\mathbf{D}\left(\mathbf{u}_{m}\right)\right)-\tilde{\mathbf{S}}\right): \nabla \boldsymbol{\phi} \mathrm{d} x \mathrm{~d} t \\
= & \int_{0}^{T} \int_{G^{\prime}}\left(\mathbf{u}_{m} \otimes \mathbf{u}_{m} \Phi_{m}\left(\left|\mathbf{u}_{m}\right|\right)-\mathbf{u} \otimes \mathbf{u}\right): \nabla \boldsymbol{\phi} \mathrm{d} x \mathrm{~d} t \\
& +\int_{0}^{T} \int_{G^{\prime}}\left(p_{1, m}+p_{2, m}\right) \operatorname{div} \boldsymbol{\phi}+\nabla p_{h, m} \cdot \partial_{t} \boldsymbol{\phi} \mathrm{d} x \mathrm{~d} t
\end{aligned}
$$

for all $\boldsymbol{\phi} \in C_{0}^{\infty}\left(G^{\prime} \times(0, T)\right)$. Moreover, by (2.4), (2.5), and $\mathbf{u}_{m}(0)-\mathbf{u}(0)=\mathbf{0}$ we have

$$
\begin{aligned}
\left\|p_{1, m}\right\|_{L^{q^{\prime}}\left(G^{\prime} \times(0, T)\right)} & \leq c\left\|\mathbf{S}\left(\cdot, \mathbf{D}\left(\mathbf{u}_{m}\right)\right)-\tilde{\mathbf{S}}\right\|_{L^{q^{\prime}}\left(G^{\prime} \times(0, T)\right)} \\
\left\|p_{2, m}\right\|_{L^{\sigma_{0}}\left(G^{\prime} \times(0, T)\right)} & \leq c\left\|\mathbf{u}_{m} \otimes \mathbf{u}_{m} \Phi_{m}\left(\left|\mathbf{u}_{m}\right|\right)-\mathbf{u} \otimes \mathbf{u}\right\|_{L^{\sigma_{0}\left(G^{\prime} \times(0, T)\right)}} \\
\left\|p_{h, m}(t)\right\|_{W^{1,2}\left(G^{\prime}\right)} & \leq c\left\|\mathbf{u}_{m}(t)-\mathbf{u}(t)\right\|_{L^{2}\left(G^{\prime}\right)}, \quad t \in(0, T)
\end{aligned}
$$

Since $p_{h, m}$ is harmonic in $G^{\prime}$, it follows by the well-known local regularity theory (cf. [21, Theorem 8.24]) and (4.20) that for all $t \in(0, T)$ and all $1 \leq r \leq \infty$

$$
\begin{aligned}
\left\|p_{h, m}(t)\right\|_{W^{2, r}(G)} & \leq c\left\|p_{h, m}(t)\right\|_{L^{2}\left(G^{\prime}\right)} \\
& \leq c\left\|\mathbf{u}_{m}(t)-\mathbf{u}(t)\right\|_{L^{2}\left(G^{\prime}\right)}
\end{aligned}
$$

where the constant depends on $n, G^{\prime}$ and $G$. From (4.13) and (4.21) with $r=2 \sigma_{0}$ it follows that

$$
\left.p_{h, m}\right|_{G \times(0, T)} \rightarrow 0 \quad \text { strongly in } \quad L^{2 \sigma_{0}}\left(0, T ; W^{2,2 \sigma_{0}}(G)\right) \quad \text { as } \quad m \rightarrow \infty
$$

Moreover, we know from (4.21) with $r=\infty$ and (4.7) that

$$
\left\|p_{h, m}\right\|_{L^{\infty}\left(0, T ; W^{2, \infty}(G)\right)} \leq c .
$$

By interpolation it follows from (4.22) and (4.23) that for all $r \in[1, \infty)$

$$
\left\|p_{h, m}\right\|_{L^{r}\left(0, T ; W^{2, r}(G)\right)} \rightarrow 0 \quad \text { as } m \rightarrow \infty .
$$


In order to use Theorem 3.9 we define

$$
\mathbf{v}_{m}:=\left(\mathbf{u}_{m}-\mathbf{u}+\nabla p_{h, m}\right) \chi_{G \times(0, T)} .
$$

This definition together with (4.13) and (4.22) implies

$$
\mathbf{v}_{m} \rightarrow \mathbf{0} \quad \text { strongly in } \quad L^{2 \sigma_{0}}(G \times(0, T)) \quad \text { as } \quad m \rightarrow \infty .
$$

Moreover, the identity (4.17) can be written for every $\varphi \in C_{0}^{\infty}(G \times(0, T))$ as

$$
\begin{aligned}
& -\int_{0}^{T} \int_{G} \mathbf{v}_{m} \cdot \partial_{t} \boldsymbol{\phi} \mathrm{d} x \mathrm{~d} t+\int_{0}^{T} \int_{G}\left(\mathbf{S}\left(\mathbf{D}\left(\mathbf{u}_{m}\right)\right)-\tilde{\mathbf{S}}\right): \nabla \boldsymbol{\phi} \mathrm{d} x \mathrm{~d} t \\
= & \int_{0}^{T} \int_{G}\left(\mathbf{u}_{m} \otimes \mathbf{u}_{m} \Phi_{m}\left(\left|\mathbf{u}_{m}\right|\right)-\mathbf{u} \otimes \mathbf{u}\right): \nabla \boldsymbol{\phi} \mathrm{d} x \mathrm{~d} t \\
& +\int_{0}^{T} \int_{G}\left(p_{1, m}+p_{2, m}\right) \operatorname{div} \boldsymbol{\phi} \mathrm{d} x \mathrm{~d} t .
\end{aligned}
$$

Using (4.11), $\mathbf{u}_{m}, \mathbf{u} \in L^{2 \sigma_{0}}(G \times(0, T))$, (4.14), (4.18), (4.19), and the properties of $\sigma_{0}$ we get from (4.26) that the weak derivative $\partial_{t} \mathbf{v}_{m}$ belongs to the space $L^{\sigma_{0}}\left(0, T ;\left(W_{0}^{1, \sigma_{0}^{\prime}}(G)\right)^{*}\right)$. Therefore setting

$$
\begin{aligned}
\mathbf{H}_{1, m} & :=\tilde{\mathbf{S}}-\mathbf{S}\left(\mathbf{D}\left(\mathbf{u}_{m}\right)\right)+p_{1, m} \mathbf{I}, \\
\mathbf{H}_{2, m} & :=\mathbf{u}_{m} \otimes \mathbf{u}_{m} \Phi_{m}\left(\left|\mathbf{u}_{m}\right|\right)-\mathbf{u} \otimes \mathbf{u}+p_{2, m} \mathbf{I}, \\
\mathbf{H}_{m} & :=\mathbf{H}_{1, m}+\mathbf{H}_{2, m}
\end{aligned}
$$

a.e. in $G \times(0, T)$ and extending to $\mathbb{R}^{n+1}$ by zero, the identity (4.26) can be written both as

$$
-\int_{G \times(0, T)} \mathbf{v}_{m} \cdot \partial_{t} \boldsymbol{\varphi} \mathrm{d} X=\int_{G \times(0, T)} \mathbf{H}_{m}: \nabla \boldsymbol{\varphi} \mathrm{d} X
$$

for all $\varphi \in C_{0}^{\infty}(G \times(0, T))$ and as

$$
\int_{0}^{T}\left\langle\partial_{t} \mathbf{v}_{m}, \boldsymbol{\varphi}\right\rangle \mathrm{d} t=\int_{G \times(0, T)} \mathbf{H}_{m}: \nabla \boldsymbol{\varphi} \mathrm{d} X
$$

for all $\boldsymbol{\varphi} \in L^{\sigma_{0}^{\prime}}\left(0, T ; W_{0}^{1, \sigma_{0}^{\prime}}(G)\right)$. Next, we set

$$
g_{m}:=\mathcal{M}^{*}\left(\left|\nabla \mathbf{v}_{m}\right|\right)+\left(\mathcal{M}^{*}\left(\left|\mathbf{H}_{1, m}\right|\right)\right)^{\frac{1}{q-1}},
$$

where $\mathcal{M}^{*}$ is defined in Appendix A. Due to (4.8), (4.10), (4.11), (4.18), and (4.21) for $r=q$ we know that $\nabla \mathbf{v}_{m}$ is uniformly bounded in $L^{q}\left(\mathbb{R}^{n+1}\right)$ and $\mathbf{H}_{1, m}$ is 
uniformly bounded in $L^{q^{\prime}}\left(\mathbb{R}^{n+1}\right)$. Now, by the boundedness of $\mathcal{M}^{*}$ from $L^{q}\left(\mathbb{R}^{n+1}\right)$ to $L^{q}\left(\mathbb{R}^{n+1}\right)$, respectively $L^{q^{\prime}}\left(\mathbb{R}^{n+1}\right)$ to $L^{q^{\prime}}\left(\mathbb{R}^{n+1}\right)$, (see Appendix A) we see that

$$
\left\|g_{m}\right\|_{L^{q}\left(\mathbb{R}^{n+1}\right)} \leq c .
$$

Thus we obtain for $k \in \mathbb{N}$

$$
\begin{aligned}
c^{q} & \geq \int_{2^{2^{k}}}^{2^{2^{k+1}}} \lambda^{q-1} \mathcal{L}_{n+1}\left(\left\{\left|g_{m}\right|>\lambda\right\}\right) \mathrm{d} \lambda \\
& \geq \int_{2^{2^{k}}}^{2^{2^{k+1}}} \lambda^{-1} \mathrm{~d} \lambda \inf _{2^{2^{k}} \leq \gamma \leq 2^{2^{k+1}}} \gamma^{q} \mathcal{L}_{n+1}\left(\left\{\left|g_{m}\right|>\gamma\right\}\right) \\
& =2^{k} \ln (2) \inf _{2^{2^{k}} \leq \gamma \leq 2^{2^{k+1}}} \gamma^{q} \mathcal{L}_{n+1}\left(\left\{\left|g_{m}\right|>\gamma\right\}\right) .
\end{aligned}
$$

Consequently there exists $\lambda_{k, m} \in\left[2^{2^{k}}, 2^{2^{k+1}}\right]$, such that

$$
\lambda_{k, m}^{q} \mathcal{L}_{n+1}\left(\left\{\left|g_{m}\right|>\lambda_{k, m}\right\}\right) \leq c^{q}(\ln 2)^{-1} 2^{-k} .
$$

We define for $k, m \in \mathbb{N}$

$$
G_{k, m}:=\left\{\left|g_{m}\right|>\lambda_{k, m}\right\}
$$

and

$$
\alpha_{k, m}:=\lambda_{k, m}^{2-q}
$$

Then by (4.30) we have

$$
\lambda_{k, m}^{q} \mathcal{L}_{n+1}\left(G_{k, m}\right) \leq c 2^{-k},
$$

and obtain

$$
\begin{aligned}
G_{k, m} & \supset\left\{\mathcal{M}^{*}\left(\left|\nabla \mathbf{v}_{m}\right|\right)>\lambda_{k, m}\right\} \cup\left\{\left(\mathcal{M}^{*}\left(\left|\mathbf{H}_{1, m}\right|\right)\right)^{\frac{1}{q-1}}>\lambda_{k, m}\right\} \\
& =\left\{\mathcal{M}^{*}\left(\left|\nabla \mathbf{v}_{m}\right|\right)>\lambda_{k, m}\right\} \cup\left\{\mathcal{M}^{*}\left(\left|\mathbf{H}_{1, m}\right|\right)>\lambda_{k, m}^{q-1}\right\} \\
& =\left\{\mathcal{M}^{*}\left(\left|\nabla \mathbf{v}_{m}\right|\right)>\lambda_{k, m}\right\} \cup\left\{\alpha_{k, m} \mathcal{M}^{*}\left(\left|\mathbf{H}_{1, m}\right|\right)>\lambda_{k, m}\right\} .
\end{aligned}
$$

To treat the term with $\mathbf{H}_{2, m}$ we define for $k, m \in \mathbb{N}$

$$
F_{k, m}:=\left\{\left(\mathcal{M}^{*}\left(\left|\mathbf{H}_{2, m}\right|\right)\right)^{\frac{1}{q-1}}>\lambda_{k, m}\right\} .
$$

Using the weak type estimate for $\mathcal{M}^{*}\left(c f\right.$. (A.5)) and $\sigma_{0}>1$ we get

$$
\begin{aligned}
\mathcal{L}_{n+1}\left(F_{k, m}\right) & =\mathcal{L}_{n+1}\left(\left\{\mathcal{M}^{*}\left(\left|\mathbf{H}_{2, m}\right|\right)>\lambda_{k, m}^{q-1}\right\}\right) \\
& \leq c \lambda_{k, m}^{(1-q) \sigma_{0}}\left\|\mathcal{M}^{*}\left(\left|\mathbf{H}_{2, m}\right|\right)\right\|_{\sigma_{0}}^{\sigma_{0}} \\
& \leq c \lambda_{k, m}^{(1-q) \sigma_{0}}\left\|\mathbf{H}_{2, m}\right\|_{\sigma_{0}}^{\sigma_{0}} .
\end{aligned}
$$


Since $\lambda_{k, m} \geq 2^{2^{k}}$ and $\mathbf{H}_{2, m}$ converges to $\mathbf{0}$ in $L^{\sigma_{0}}(G \times(0, T))$ due to (4.14) and (4.19), we obtain that for each $k \in \mathbb{N}$ there holds

$$
\limsup _{m \rightarrow \infty} \mathcal{L}_{n+1}\left(F_{k, m}\right)=0
$$

Analogously to (4.32) we get

$$
F_{k, m}=\left\{\alpha_{k, m} \mathcal{M}^{*}\left(\left|\mathbf{H}_{2, m}\right|\right)>\lambda_{k, m}\right\} .
$$

Finally we define for $k, m \in \mathbb{N}$

$$
H_{k, m}:=\left\{\mathcal{M}^{*}\left(\left|\mathbf{v}_{m}\right|\right)>1\right\} .
$$

Using the weak type estimate for $\mathcal{M}^{*}\left(c f\right.$. (A.5)) and $\sigma_{0}>1$ we get

$$
\begin{aligned}
\mathcal{L}_{n+1}\left(H_{k, m}\right) & \left.=\mathcal{L}_{n+1}\left\{\mathcal{M}^{*}\left(\left|\mathbf{v}_{m}\right|\right)>1\right\}\right) \\
& \leq c\left\|\mathcal{M}^{*}\left(\left|\mathbf{v}_{m}\right|\right)\right\|_{2 \sigma_{0}}^{2 \sigma_{0}} \\
& \leq c\left\|\mathbf{v}_{m}\right\|_{2 \sigma_{0}}^{2 \sigma_{0}} .
\end{aligned}
$$

Since $\mathbf{v}_{m}$ converges to $\mathbf{0}$ in $L^{\sigma_{0}}(G \times(0, T))$ due to (4.25), we obtain that for each $k \in \mathbb{N}$ there holds

$$
\limsup _{m \rightarrow \infty} \mathcal{L}_{n+1}\left(H_{k, m}\right)=0
$$

Since $\mathcal{M}^{*}$ is subadditive and $\mathbf{H}_{m}=\mathbf{H}_{1, m}+\mathbf{H}_{2, m}$, (4.35) and (4.32) imply

$$
\begin{aligned}
G_{k, m} \cup F_{k, m} & \supset\left\{\mathcal{M}^{*}\left(\left|\nabla \mathbf{v}_{m}\right|\right)>\lambda_{k, m}\right\} \cup\left\{\alpha_{k, m} \mathcal{M}^{*}\left(\left|\mathbf{H}_{m}\right|\right)>2 \lambda_{k, m}\right\} \\
& \supset\left\{\mathcal{M}^{*}\left(\left|\nabla \mathbf{v}_{m}\right|\right)+\alpha_{k, m} \mathcal{M}^{*}\left(\left|\mathbf{H}_{m}\right|\right)>3 \lambda_{k, m}\right\} .
\end{aligned}
$$

Defining

$$
E_{k, m}:=\left(G_{k, m} \cup F_{k, m} \cup H_{k, m}\right) \cap(G \times(0, T))
$$

we see that $E_{k, m}$ satisfies the assumption (3.14) of Theorem 3.9 with $\Lambda=3 \lambda_{k, m}$. Let us fix a cut-off function $\zeta \in C_{0}^{\infty}(G \times(0, T))$ with $0 \leq \zeta \leq 1$ in $G \times(0, T)$. In view of (4.28), (4.38) and (4.39) we are able to apply Theorem 3.9 with $K:=\operatorname{supp}(\zeta), \mathbf{u}=\mathbf{v}_{m}, \mathbf{H}=\mathbf{H}_{m}, E=E_{k, m}$, and $\alpha=\alpha_{k, m}$. We denote $\mathcal{T}_{k, m}:=\mathcal{T}_{E_{k, m}}^{\alpha_{k, m}}$. Hence, inserting the admissible test function $\boldsymbol{\varphi}=\zeta \mathcal{T}_{k, m} \mathbf{v}_{m}$ into 
(4.29), using (4.27) and taking into account (3.18) yields

$$
\begin{aligned}
& \int_{G \times(0, T)}\left(\mathbf{S}\left(\mathbf{D}\left(\mathbf{u}_{m}\right)\right)-\tilde{\mathbf{S}}\right): \mathbf{D}\left(\mathcal{T}_{k, m} \mathbf{v}_{m}\right) \zeta \mathrm{d} X \\
& =\int_{G \times(0, T)}\left(\tilde{\mathbf{S}}-\mathbf{S}\left(\mathbf{D}\left(\mathbf{u}_{m}\right)\right)\right):\left(\mathcal{T}_{k, m} \mathbf{v}_{m} \otimes \nabla \zeta\right) \mathrm{d} X \\
& \quad+\int_{G \times(0, T)}\left(\mathbf{u} \otimes \mathbf{u}-\mathbf{u}_{m} \otimes \mathbf{u}_{m} \Phi_{m}\left(\left|\mathbf{u}_{m}\right|\right)\right): \nabla\left(\left(\mathcal{T}_{k, m} \mathbf{v}_{m}\right) \zeta\right) \mathrm{d} X \\
& \quad+\int_{G \times(0, T)} p_{1, m} \mathcal{T}_{k, m} \mathbf{v}_{m} \cdot \nabla \zeta \mathrm{d} X \\
& \quad+\int_{G \times(0, T)} p_{1, m} \operatorname{div}\left(\mathcal{T}_{k, m} \mathbf{v}_{m}\right) \zeta \mathrm{d} X \\
& \quad+\int_{G \times(0, T)} p_{2, m} \operatorname{div}\left(\left(\mathcal{T}_{k, m} \mathbf{v}_{m}\right) \zeta\right) \mathrm{d} X \\
& \quad+\frac{1}{2} \int_{G \times(0, T)}\left(2 \mathbf{v}_{m} \cdot \mathcal{T}_{k, m} \mathbf{v}_{m}-\left|\mathcal{T}_{k, m} \mathbf{v}_{m}\right|^{2}\right) \partial_{t} \zeta \mathrm{d} X \\
& \quad+\int_{E_{k, m}}\left(\partial_{t} \mathcal{T}_{k, m} \mathbf{v}_{m}\right) \cdot\left(\mathbf{v}_{m}-\mathcal{T}_{k, m} \mathbf{v}_{m}\right) \zeta \mathrm{d} X \\
& =I_{m}^{k}+I I_{m}^{k}+I I I_{m}^{k}+I V_{m}^{k}+V_{m}^{k}+V I_{m}^{k}+V I I_{m}^{k} .
\end{aligned}
$$

Next, for a fixed $k \in \mathbb{N}$ we will carry out the passage to the limit $m \rightarrow \infty$ in all integrals $I_{m}^{k}, \ldots, V I I_{m}^{k}$ separately.

(i) $\lim \sup _{m \rightarrow \infty}\left(\left|I_{m}^{k}\right|+\left|I I I_{m}^{k}\right|\right)=0$.

Due to (4.10) and (4.11) we have that $\mathbf{S}\left(\mathbf{D}\left(\mathbf{u}_{m}\right)\right)$ and $\tilde{\mathbf{S}}$ are uniformly bounded in $L^{q^{\prime}}(G \times(0, T))$. Using this, Hölder's inequality and (3.7) one obtains

$$
\left|I_{m}^{k}\right| \leq c\left(\int_{G \times(0, T)}\left|\left(\mathcal{T}_{k, m} \mathbf{v}_{m}\right) \otimes \nabla \zeta\right|^{q} \mathrm{~d} X\right)^{\frac{1}{q}} \leq c\left\|\mathbf{v}_{m}\right\|_{L^{q}(G \times(0, T))} .
$$

Thus $\lim \sup _{m \rightarrow \infty}\left|I_{m}^{k}\right|=0$ follows with the aid of (4.25) and $2 \sigma_{0} \geq q$. Arguing similarly one verifies $\lim \sup _{m \rightarrow \infty}\left|I I I_{m}^{k}\right|=0$. Hereby, we use that $p_{1, m}$ is uniformly bounded in $L^{q^{\prime}}(G \times(0, T))$ due to (4.18), (4.10) and (4.11).

(ii) $\limsup _{m \rightarrow \infty}\left(\left|I I_{m}^{k}\right|+\left|V_{m}^{k}\right|\right)=0$.

We estimate

$$
\left|I I_{m}^{k}\right| \leq c\left\|\mathbf{u} \otimes \mathbf{u}-\mathbf{u}_{m} \otimes \mathbf{u}_{m} \Phi_{m}\left(\left|\mathbf{u}_{m}\right|\right)\right\|_{L^{1}(G \times(0, T))}\left\|\nabla\left(\mathcal{T}_{k, m} \mathbf{v}_{m} \zeta\right)\right\|_{L^{\infty}(K)} .
$$

It follows from (4.14), $\sigma_{0}>1$, and $G$ being bounded that

$$
\limsup _{m \rightarrow \infty}\left\|\mathbf{u} \otimes \mathbf{u}-\mathbf{u}_{m} \otimes \mathbf{u}_{m} \Phi_{m}\left(\left|\mathbf{u}_{m}\right|\right)\right\|_{L^{1}(G \times(0, T))}=0 .
$$


Thus we need to show that for fixed $k \in \mathbb{N}$ the sequence $\nabla\left(\mathcal{T}_{k, m} \mathbf{v}_{m} \zeta\right)$ is uniformly bounded in $L^{\infty}(\operatorname{supp}(\zeta))$ with respect to $m$. This and (4.41) then imply $\limsup _{m \rightarrow \infty}\left|I I_{m}^{k}\right|=0$. For fixed $k$ the sequence $\lambda_{k, m}$ lies in the interval $\left[2^{2^{k}}, 2^{2^{k+1}}\right]$ and thus $\alpha_{k, m}=\lambda_{k, m}^{2-q}$ is uniformly bounded from above. This implies that for fixed $k$ holds

$$
\inf _{m \in \mathbb{N}} \delta_{\alpha_{k, m}, K}>0
$$

So according to (3.15) and (3.16) we get for fixed $k \in \mathbb{N}$ and all $m \in \mathbb{N}$

$$
\begin{aligned}
\left\|\nabla\left(\mathcal{T}_{k, m} \mathbf{v}_{m} \zeta\right)\right\|_{L^{\infty}(K)} \leq & \left\|\nabla \mathcal{T}_{k, m} \mathbf{v}_{m}\right\|_{L^{\infty}(K)}+c(\nabla \zeta)\left\|\mathcal{T}_{k, m} \mathbf{v}_{m}\right\|_{L^{\infty}(K)} \\
\leq & c\left(\lambda_{k, m}+\alpha_{k, m}^{-1} \delta_{\alpha_{k, m}, K}^{-n-3}\left\|\mathbf{v}_{m}\right\|_{L^{1}(E)}\right) \\
& +c\left(1+\alpha_{k, m}^{-1} \delta_{\alpha_{k, m}, K}^{-n-2}\left\|\mathbf{v}_{m}\right\|_{L^{1}(E)}\right)
\end{aligned}
$$

The uniform boundedness of $\mathbf{v}_{m}$ in $L^{1}(G \times(0, T))(c f .(4.25))$, (4.42) and $\lambda_{k, m} \in\left[2^{2^{k}}, 2^{2^{k+1}}\right]$ yield that for fixed $k \in \mathbb{N}$ the sequence $\nabla\left(\mathcal{T}_{k, m} \mathbf{v}_{m} \zeta\right)$ is uniformly uniformly bounded in $L^{\infty}(K)$ with respect to $m$. This proves $\limsup _{m \rightarrow \infty}\left|I I_{m}^{k}\right|=0$.

Using (4.19) and (4.41) we can prove analogously $\lim \sup _{m \rightarrow \infty}\left|V_{m}^{k}\right|=0$.

(iii) $\limsup _{m \rightarrow \infty}\left|V I_{m}^{k}\right|=0$.

Using Cauchy-Schwarz's inequality and (3.7) shows that

$$
\left|V I_{m}^{k}\right| \leq c\left\|\mathbf{v}_{m}\right\|_{L^{2}(G \times(0, T))}^{2} .
$$

Since $\sigma_{0}>1$, the assertion follows from (4.25).

(iv) $\limsup _{m \rightarrow \infty}\left|I V_{m}^{k}\right| \leq c 2^{-k / q}$.

Since $\operatorname{div} \mathbf{u}_{m}=\operatorname{div} \mathbf{u}=\Delta p_{h, m}=0$ on the set $G^{\prime} \times(0, T)$ we have $\operatorname{div} \mathbf{v}_{m}=0$ on this set. From this, the definition of $\mathcal{T}_{k, m}$ and (3.29) applied to $\mathcal{T}_{k, m}$ we obtain that $\operatorname{div} \mathcal{T}_{k, m} \mathbf{v}_{m}=\operatorname{div} \mathbf{v}_{m}=0$ holds on the set $(G \times(0, T)) \backslash E_{k, m}$. Thus, we have

$$
I V_{m}^{k}=\int_{G \times(0, T)} p_{1, m} \operatorname{div}\left(\mathcal{T}_{k, m} \mathbf{v}_{m}\right) \zeta \mathrm{d} X=\int_{E_{k, m} \cap K} p_{1, m} \operatorname{div}\left(\mathcal{T}_{k, m} \mathbf{v}_{m}\right) \zeta \mathrm{d} X
$$

Hölder's inequality implies

$$
\left|I V_{m}^{k}\right| \leq\left\|p_{1, m}\right\|_{L^{q^{\prime}}(G \times(0, T))}\left\|\nabla\left(\mathcal{T}_{k, m} \mathbf{v}_{m}\right)\right\|_{L^{q}\left(E_{k, m} \cap K\right)} .
$$

From (4.18), (4.11) and (4.10) one finds

$$
\left\|p_{1, m}\right\|_{L^{q^{\prime}}(G \times(0, T))} \leq c \| \mathbf{S}\left(\mathbf{D}\left(\mathbf{u}_{m}\right)-\tilde{\mathbf{S}} \|_{L^{q^{\prime}}(G \times(0, T))} \leq c .\right.
$$


Therefore we obtain with (3.15), (4.42), $\lambda_{k, m} \in\left[2^{2^{k}}, 2^{2^{k+1}}\right], \sigma_{0}>1$, and (4.25) that

$$
\begin{aligned}
\limsup _{m \rightarrow \infty}\left|I V_{m}^{k}\right| & \leq c \limsup _{m \rightarrow \infty}\left\|\nabla\left(\mathcal{T}_{k, m} \mathbf{v}_{m}\right)\right\|_{L^{q}\left(E_{k, m} \cap K\right)} \\
& \leq c \limsup _{m \rightarrow \infty}\left(\mathcal{L}_{n+1}\left(E_{k, m}\right)^{1 / q}\left(\lambda_{k, m}+\frac{\left\|\mathbf{v}_{m}\right\|_{L^{1}\left(E_{k, m}\right)}}{\alpha_{k, m} \delta_{\alpha_{k, m}, K}^{n+3}}\right)\right) \\
& \leq c \limsup _{m \rightarrow \infty}\left(\mathcal{L}_{n+1}\left(E_{k, m}\right)^{1 / q}\left(\lambda_{k, m}+\frac{\left\|\mathbf{v}_{m}\right\|_{L^{1}(G \times(0, T))}}{\alpha_{k, m} \delta_{\alpha_{k, m}, K}^{n+3}}\right)\right) \\
& =c \limsup _{m \rightarrow \infty}\left(\mathcal{L}_{n+1}\left(E_{k, m}\right)^{1 / q} \lambda_{k, m}\right) .
\end{aligned}
$$

From the definition of $E_{k, m}$ follows

$$
\mathcal{L}_{n+1}\left(E_{k, m}\right) \leq \mathcal{L}_{n+1}\left(G_{k, m}\right)+\mathcal{L}_{n+1}\left(F_{k, m}\right)+\mathcal{L}_{n+1}\left(H_{k, m}\right)
$$

This, (4.31), (4.34) and (4.37) imply

$$
\limsup _{m \rightarrow \infty}\left(\mathcal{L}_{n+1}\left(E_{k, m}\right)^{1 / q} \lambda_{k, m}\right) \leq c 2^{-k / q}
$$

From (4.43) and (4.44) follows the claim.

(v) $\lim \sup _{m \rightarrow \infty}\left|V I I_{m}^{k}\right| \leq c 2^{-k}$.

From the definition of $\alpha_{k, m},(3.17)$ and $E_{k, m} \subset G \times(0, T)$ follows

$$
\left|V I I_{m}^{k}\right| \leq c \mathcal{L}_{n+1}\left(E_{k, m}\right) \lambda_{k, m}^{q-2}\left(\lambda_{k, m}+\frac{\left\|\mathbf{v}_{m}\right\|_{L^{1}(G \times(0, T))}}{\alpha_{k, m} \delta_{\alpha_{k, m}, K}^{n+3}}\right)^{2}
$$

Using $\lambda_{k, m} \in\left[2^{2^{k}}, 2^{2^{k+1}}\right],(4.42), \sigma_{0}>1$, and (4.25) and (4.44) we get

$$
\limsup _{m \rightarrow \infty}\left|V I I_{m}^{k}\right| \leq c \limsup _{m \rightarrow \infty}\left(\mathcal{L}_{n+1}\left(E_{k, m}\right) \lambda_{k, m}^{q}\right) \leq c 2^{-k}
$$

which proves the claim.

Altogether, we proved in (i)-(v) that for each fixed $k \in \mathbb{N}$

$$
\limsup _{m \rightarrow \infty}\left|\int_{G \times(0, T)}\left(\mathbf{S}\left(\mathbf{D}\left(\mathbf{u}_{m}\right)\right)-\tilde{\mathbf{S}}\right): \mathbf{D}\left(\mathcal{T}_{k, m} \mathbf{v}_{m}\right) \zeta \mathrm{d} X\right| \leq c 2^{-k / q}
$$

where we used $1<q$. 
Arguing similarly as in (iv) one easily proves using (4.10), (4.11), (4.43) and (4.44) that

$$
\begin{aligned}
& \limsup _{m \rightarrow \infty}\left|\int_{E_{k, m}}\left(\mathbf{S}\left(\mathbf{D}\left(\mathbf{u}_{m}\right)\right)-\tilde{\mathbf{S}}\right): \mathbf{D}\left(\mathcal{T}_{k, m} \mathbf{v}_{m}\right) \zeta \mathrm{d} X\right| \\
& \leq \limsup _{m \rightarrow \infty}\left(\| \tilde{\mathbf{S}}-\mathbf{S}\left(\mathbf{D}\left(\mathbf{u}_{m}\right)\left\|_{L^{q^{\prime}}(G \times(0, T))}\right\| \nabla\left(\mathcal{T}_{k, m} \mathbf{v}_{m}\right) \|_{L^{q}\left(E_{k, m}\right)}\right)\right. \\
& \leq c \limsup _{m \rightarrow \infty}\left(\lambda_{k, m} \mathcal{L}_{n+1}\left(E_{k, m}\right)^{1 / q}\right) \\
& \leq c 2^{-k / q} .
\end{aligned}
$$

Due to (3.29) on the set $(G \times(0, T)) \backslash E_{k, m}$ holds $\mathbf{D}\left(\mathcal{T}_{k, m} \mathbf{v}_{m}\right)=\mathbf{D}\left(\mathbf{v}_{m}\right)$. Thus we obtain from (4.45) and (4.46)

$$
\limsup _{m \rightarrow \infty}\left|\int_{(G \times(0, T)) \backslash E_{k, m}}\left(\mathbf{S}\left(\mathbf{D}\left(\mathbf{u}_{m}\right)\right)-\tilde{\mathbf{S}}\right): \mathbf{D}\left(\mathbf{v}_{m}\right) \zeta \mathrm{d} X\right| \leq c 2^{-k / q} .
$$

Recall that $\mathbf{v}_{m}=\mathbf{u}_{m}-\mathbf{u}-\nabla p_{h, m}$. Using (4.10), (4.11), (4.24) and $q \leq 2 \sigma_{0}$ we obtain

$$
\lim _{m \rightarrow \infty} \int_{(G \times(0, T)) \backslash E_{k, m}}\left(\mathbf{S}\left(\mathbf{D}\left(\mathbf{u}_{m}\right)\right)-\tilde{\mathbf{S}}\right): \mathbf{D}\left(\nabla p_{h, m}\right) \zeta \mathrm{d} X=0 .
$$

This and (4.47) yields for each fixed $k \in \mathbb{N}$

$$
\limsup _{m \rightarrow \infty}\left|\int_{(G \times(0, T)) \backslash E_{k, m}}\left(\mathbf{S}\left(\mathbf{D}\left(\mathbf{u}_{m}\right)\right)-\tilde{\mathbf{S}}\right): \mathbf{D}\left(\mathbf{u}_{m}-\mathbf{u}\right) \zeta \mathrm{d} X\right| \leq c 2^{-k / q} .
$$

Due to (4.34), (4.37) and (4.48) we can find for each $k \in \mathbb{N}$ a number $m_{k} \in \mathbb{N}$, such that

$$
\begin{aligned}
& \left|\int_{(G \times(0, T)) \backslash E_{k, m_{k}}}\left(\mathbf{S}\left(\mathbf{D}\left(\mathbf{u}_{m_{k}}\right)\right)-\tilde{\mathbf{S}}\right): \mathbf{D}\left(\mathbf{u}_{m_{k}}-\mathbf{u}\right) \zeta \mathrm{d} X\right| \leq c 2^{-k / q}, \\
& \mathcal{L}_{n+1}\left(F_{k, m_{k}}\right) \leq c 2^{-k}, \\
& \mathcal{L}_{n+1}\left(H_{k, m_{k}}\right) \leq c 2^{-k} \text {. }
\end{aligned}
$$

Setting $\zeta_{k}:=\zeta \chi(G \times(0, T)) \backslash E_{k, m_{k}}(k \in \mathbb{N})$ one gets

$$
\zeta_{k} \rightarrow \zeta \quad \text { a.e. in } G \times(0, T) \text { as } \quad k \rightarrow \infty .
$$

Indeed, there holds

$$
\zeta_{k}(X) \rightarrow \zeta(X) \quad \forall X \in \bigcup_{k=1}^{\infty} \bigcap_{\ell=k}^{\infty}\left((G \times(0, T)) \backslash E_{\ell, m_{\ell}}\right) \quad \text { as } \quad k \rightarrow \infty
$$


We have $\bigcup_{k=1}^{\infty} \bigcap_{\ell=k}^{\infty}\left((G \times(0, T)) \backslash E_{\ell, m_{\ell}}\right)=(G \times(0, T)) \backslash \bigcap_{k=1}^{\infty} \bigcup_{\ell=k}^{\infty} E_{\ell, m_{\ell}}$. Moreover, due to (4.31), $\lambda_{k, m} \geq 1$ and (4.49) we get for all $k \in \mathbb{N}$

$$
\mathcal{L}_{n+1}\left(\bigcup_{\ell=k}^{\infty} E_{\ell, m_{\ell}}\right) \leq \sum_{\ell=k}^{\infty}\left(\mathcal{L}_{n+1}\left(G_{\ell, m_{\ell}}\right)+\mathcal{L}_{n+1}\left(F_{\ell, m_{\ell}}\right)+\mathcal{L}_{n+1}\left(H_{\ell, m_{\ell}}\right)\right) \leq c 2^{-k},
$$

which implies

$$
\mathcal{L}_{n+1}\left(\bigcap_{k=1}^{\infty} \bigcup_{\ell=k}^{\infty} E_{\ell, m_{\ell}}\right)=0
$$

Whence, (4.50) holds.

From (4.50), (4.11) and the Lebesgue Theorem on dominated convergence we get that $\tilde{\mathbf{S}} \zeta_{k}$ converges to $\tilde{\mathbf{S}} \zeta$ strongly in $L^{q^{\prime}}(G \times(0, T))$, and $\mathbf{D}(\mathbf{u}) \zeta_{k}$ converges to $\mathbf{D}(\mathbf{u}) \zeta$ strongly in $L^{q}(G \times(0, T))$. Thus from (4.49) and (4.11) we deduce

$$
\lim _{k \rightarrow \infty} \int_{G \times(0, T)} \mathbf{S}\left(\mathbf{D}\left(\mathbf{u}_{m_{k}}\right)\right): \mathbf{D}\left(\mathbf{u}_{m_{k}}\right) \zeta_{k} \mathrm{~d} X=\int_{G \times(0, T)} \tilde{\mathbf{S}}: \mathbf{D}(\mathbf{u}) \zeta \mathrm{d} X .
$$

With help of the local Minty trick ( $c f$. [52, Lemma A.2]) we obtain

$$
\tilde{\mathbf{S}} \zeta=\mathbf{S}(\cdot, \mathbf{D}(\mathbf{u})) \zeta \quad \text { a.e. in } \quad G \times(0, T) .
$$

This concludes the proof of the theorem.

\section{Appendix}

\section{A. Maximal operators}

In this appendix we recall some results regarding maximal operators.

For $g \in L_{\text {loc }}^{1}\left(\mathbb{R}^{n+1}\right)$ we define for $(x, t) \in \mathbb{R}^{n+1}$

$$
\begin{aligned}
& \mathcal{M}_{x}(g)(x, t):=\sup _{0<r<\infty} f_{B_{r}(x)}|g(y, t)| \mathrm{d} y, \\
& \mathcal{M}_{t}(g)(x, t):=\sup _{0<\rho<\infty} f_{I_{\rho}(t)}|g(x, s)| \mathrm{d} s,
\end{aligned}
$$

where $I_{\rho}(t)$ denotes the interval $(t-\rho, t+\rho)$. Clearly, for $g \in L^{p}\left(\mathbb{R}^{n+1}\right)$, $1<p<\infty$, there exists $N \subset \mathbb{R}$ with $\mathcal{L}_{1}(N)=0$, such that $g(\cdot, t) \in L^{p}\left(\mathbb{R}^{n}\right)$ for all $t \in \mathbb{R} \backslash N$. The properties of the maximal operator (cf. [48]) yield $\mathcal{M}_{x}(g)(\cdot, t) \in$ $L^{p}\left(\mathbb{R}^{n}\right), 1<p<\infty$, for all $t \in \mathbb{R} \backslash N$ and

$$
\int_{\mathbb{R}^{n}}\left(\mathcal{M}_{x}(g)(x, t)\right)^{p} \mathrm{~d} x \leq c \int_{\mathbb{R}^{n}}|g(x, t)|^{p} \mathrm{~d} x \quad \forall t \in \mathbb{R} \backslash N .
$$


Hence, it follows that $\mathcal{M}_{x}(g) \in L^{p}\left(\mathbb{R}^{n+1}\right)$ and we have

$$
\left\|\mathcal{M}_{x}(g)\right\|_{L^{p}\left(\mathbb{R}^{n+1}\right)} \leq c\|g\|_{L^{p}\left(\mathbb{R}^{n+1}\right)} .
$$

By an analogous reasoning one obtains $\mathcal{M}_{t}(g) \in L^{p}\left(\mathbb{R}^{n+1}\right)$ and there holds

$$
\left\|\mathcal{M}_{t}(g)\right\|_{L^{p}\left(\mathbb{R}^{n+1}\right)} \leq c\|g\|_{L^{p}\left(\mathbb{R}^{n+1}\right)} .
$$

Next, we define

$$
\mathcal{M}^{*}(g):=\mathcal{M}_{t}\left(\mathcal{M}_{x}(g)\right), \quad g \in L^{p}\left(\mathbb{R}^{n+1}\right) .
$$

With help of (A.1) and (A.2) we verify the strong type estimate

$$
\left\|\mathcal{M}^{*}(g)\right\|_{L^{p}\left(\mathbb{R}^{n+1}\right)} \leq c\|g\|_{L^{p}\left(\mathbb{R}^{n+1}\right)},
$$

which implies the weak type estimate

$$
\mathcal{L}_{n+1}\left(\left\{\mathcal{M}^{*}(g)>\lambda\right\}\right) \leq c \lambda^{-p}\|g\|_{L^{p}\left(\mathbb{R}^{n+1}\right)}^{p},
$$

Moreover, we have for all $(x, t) \in \mathbb{R}^{n+1}$ and $r, \rho>0$

$$
f_{I_{\rho}(t)} f_{B_{r}(x)}|g(y, s)| \mathrm{d} y \mathrm{~d} s \leq \mathcal{M}^{*}(g)(x, t) .
$$

Indeed, for $(x, t) \in \mathbb{R}^{n+1}$ one estimates using the definition of $\mathcal{M}_{x}\left(\mathcal{M}_{t}\right.$ respectively)

$$
\begin{aligned}
f_{I_{\rho}(t)} f_{B_{r}(x)} g(y, s) \mathrm{d} y \mathrm{~d} s & \leq f_{I_{\rho}(t)} \mathcal{M}_{x}(g)(x, s) \mathrm{d} s \\
& \leq \mathcal{M}_{t}\left(\mathcal{M}_{x}(g)\right)(x, t)=\mathcal{M}^{*}(g)(x, t) .
\end{aligned}
$$

\section{B. Poincaré-type inequality}

For reader's convenience we will present a short proof of the Poincaré-type inequality we have used in the sequel of the paper.

Theorem B.1. Let for some $X_{0} \in \mathbb{R}^{n+1}$ and $0<r<\infty$ be $\mathbf{u} \in L^{1}\left(Q_{r}^{\alpha}\left(X_{0}\right)\right)$ with $\nabla \mathbf{u} \in L^{1}\left(Q_{r}^{\alpha}\left(X_{0}\right)\right)$ and $\mathbf{H} \in L^{1}\left(Q_{r}^{\alpha}\left(X_{0}\right)\right)$, where $0<\alpha<\infty$, such that

$$
-\int_{Q_{r}^{\alpha}\left(X_{0}\right)} \mathbf{u} \cdot \partial_{t} \boldsymbol{\varphi} \mathrm{d} x \mathrm{~d} t=\int_{Q_{r}^{\alpha}\left(X_{0}\right)} \mathbf{H}: \nabla \boldsymbol{\varphi} \mathrm{d} x \mathrm{~d} t \quad \forall \boldsymbol{\varphi} \in C_{0}^{\infty}\left(Q_{r}^{\alpha}\left(X_{0}\right)\right) .
$$

Then

$$
\int_{Q_{r}^{\alpha}\left(X_{0}\right)}\left|\mathbf{u}-\mathbf{u}_{Q_{r}^{\alpha}}\right| \mathrm{d} x \mathrm{~d} t \leq c_{0} r \int_{Q_{r}^{\alpha}\left(X_{0}\right)}|\nabla \mathbf{u}|+\alpha|\mathbf{H}| \mathrm{d} x \mathrm{~d} t,
$$

where $c_{0}=$ const $>0$ depends on $n$ only. 
Proof. This theorem can be found in [51, Lemma B.3] and is based on [38] and [49]. For the convenience of the reader we sketch the proof here. First we prove the assertion for $X_{0}=0, \alpha=1$ and $r=1$. For notational simplicity we write $Q_{1}$ instead of $Q_{1}^{1}(0)$ and $B_{1}$ instead of $B_{1}(0)$. For a given function $\zeta \in C_{0}^{\infty}\left(B_{1}\right)$ with $0 \leq \zeta \leq 1$ in $B_{1}$ and $\zeta \neq 0$ we define the functional $F \in L^{1}\left(B_{1}\right)^{*}$ by means of

$$
\langle F, \mathbf{v}\rangle:=\frac{1}{\int_{B_{1}} \zeta \mathrm{d} x} \int_{B_{1}} \zeta \mathbf{v} \mathrm{d} x, \quad \mathbf{v} \in L^{1}\left(B_{1}\right)
$$

As it is readily seen there holds

$$
\langle F, \mathbf{1}\rangle=1,
$$

which implies

$$
\langle F, \mathbf{v}-\langle F, \mathbf{v}\rangle\rangle=0 \quad \forall \mathbf{v} \in L^{1}\left(B_{1}(0)\right) .
$$

Thus (cf. [40, Theorem 7.1])

$$
\||\mathbf{v}|\|:=\|\nabla \mathbf{v}\|_{L^{1}\left(B_{1}\right)}+|\langle F, \mathbf{v}\rangle|
$$

defines an equivalent norm on $W^{1,1}\left(B_{1}\right)$. Thus there exists a constant $c_{n}$, such that

$$
\|\mathbf{v}-\langle F, v\rangle\|_{L^{1}\left(B_{1}\right)} \leq c_{n}\|\nabla \mathbf{v}\|_{L^{1}\left(B_{1}\right)} \quad \forall \mathbf{v} \in W^{1,1}\left(B_{1}\right) .
$$

Now let $\mathbf{u} \in L^{1}\left(Q_{1}\right)$ fulfill the assunptions of the theorem. One easily calculates

$$
\begin{aligned}
\mathbf{u}(x, t)-\mathbf{u}_{Q_{1}} & =(\mathbf{u}(x, t)-\langle F, \mathbf{u}(t)\rangle)+\left(\langle F, \mathbf{u}(t)\rangle-\mathbf{u}_{Q_{1}}\right) \\
& =(\mathbf{u}(x, t)-\langle F, \mathbf{u}(t)\rangle)+f_{Q_{1}}(\langle F, \mathbf{u}(t)\rangle-\mathbf{u}(y, s)) \mathrm{d} y \mathrm{~d} s
\end{aligned}
$$

for almost all $(x, t) \in Q_{1}$. Furthermore, one finds

$$
\begin{aligned}
& f_{Q_{1}}(\langle F, u(t)\rangle-u(y, s)) \mathrm{d} y \mathrm{~d} s \\
& =\frac{1}{\int_{B_{1}} \zeta \mathrm{d} y} f_{Q_{1}} \int_{B_{1}} \zeta\left(y^{\prime}\right)\left(u\left(y^{\prime}, t\right)-u(y, s)\right) \mathrm{d} y^{\prime} \mathrm{d} y \mathrm{~d} s \\
& =\frac{1}{2} \int_{-1}^{1}\langle F, u(t)-u(s)\rangle \mathrm{d} s \\
& \quad+\frac{1}{2 \int_{B_{1}} \zeta \mathrm{d} y} \int_{-1}^{1} f_{B_{1}} \int_{B_{1}} \zeta\left(y^{\prime}\right)\left(u\left(y^{\prime}, s\right)-u(y, s)\right) \mathrm{d} y^{\prime} \mathrm{d} y \mathrm{~d} s .
\end{aligned}
$$


The last two equalities yield

$$
\begin{aligned}
\left|\mathbf{u}(x, t)-\mathbf{u}_{Q_{1}}\right| \leq & |(\mathbf{u}(x, t)-\langle F, \mathbf{u}(t)\rangle)|+\frac{1}{2} \int_{-1}^{1}|\langle F, \mathbf{u}(t)-\mathbf{u}(s)\rangle| \mathrm{d} s \\
& +\frac{1}{2 \int_{B_{1}} \zeta \mathrm{d} y} \int_{-1}^{1} \int_{B_{1}}\left|\mathbf{u}(y, s)-\mathbf{u}(s)_{B_{1}}\right| \mathrm{d} y \mathrm{~d} s
\end{aligned}
$$

for almost all $(x, t) \in Q_{1}$. Integrating both sides of the last inequality over $Q_{1}$, estimating the first integral with the aid of (B.3), and estimating the last integral by the usual Poincaré inequality implies

$$
\begin{aligned}
& \int_{Q_{1}}\left|\mathbf{u}(x, t)-\mathbf{u}_{Q_{1}}\right| \mathrm{d} x \mathrm{~d} t \\
& \leq c \int_{Q_{1}}|\nabla \mathbf{u}| \mathrm{d} x \mathrm{~d} t+\frac{\left|B_{1}\right|}{2} \int_{-1}^{1} \int_{-1}^{1}|\langle F, \mathbf{u}(t)-\mathbf{u}(s)\rangle| \mathrm{d} s \mathrm{~d} t .
\end{aligned}
$$

Using in (B.1) the test function $\varphi(x, t)=\zeta(x) \gamma_{-h}(t)$, where $\gamma \in C_{0}^{\infty}(-1,1)$, $0 \leq \gamma \leq 1$ and $\gamma_{h}$ is the Steklov average (cf. (3.31)) we obtain

$$
\int_{Q_{1}} \partial_{t} \mathbf{u}_{h} \cdot \zeta \mathrm{d} x \mathrm{~d} t=\int_{Q_{1}} \mathbf{H}_{h}: \nabla \zeta \mathrm{d} x \mathrm{~d} t,
$$

from which one derives by standard arguments that for almost all $t \in(-1,1)$ holds for all $\zeta \in C_{0}^{\infty}\left(B_{1}\right)$

$$
\int_{B_{1}} \partial_{t} \mathbf{u}_{h}(t) \cdot \zeta \mathrm{d} x=\int_{B_{1}} \mathbf{H}_{h}(t): \nabla \zeta \mathrm{d} x .
$$

This and the definition of $F$ implies for almost all $t, s \in(-1,1)$

$$
\begin{aligned}
\left|\left\langle F, \mathbf{u}_{h}(t)-\mathbf{u}_{h}(s)\right\rangle\right| & =\left|\frac{1}{\int_{B_{1}} \zeta \mathrm{d} y} \int_{s}^{t} \int_{B_{1}} \partial_{t} \mathbf{u}_{h}(x, \tau) \cdot \zeta(x) \mathrm{d} x \mathrm{~d} \tau\right| \\
& =\left|\frac{1}{\int_{B_{1}} \zeta \mathrm{d} y} \int_{s}^{t} \int_{B_{1}} \mathbf{H}_{h}(x, \tau): \nabla \zeta(x) \mathrm{d} x \mathrm{~d} \tau\right| .
\end{aligned}
$$

The properties of the Steklov average imply for $h \rightarrow 0$

$$
|\langle F, \mathbf{u}(t)-\mathbf{u}(s)\rangle| \leq c \int_{Q_{1}}|\mathbf{H}| \mathrm{d} x \mathrm{~d} t
$$

for almost all $s, t \in(-1,1)$. This and (B.4) yield (B.2) in the special case, i.e.

$$
\int_{Q_{1}}\left|\mathbf{u}(x, t)-\mathbf{u}_{Q_{1}}\right| \mathrm{d} x \mathrm{~d} t \leq c \int_{Q_{1}}|\nabla \mathbf{u}|+|\mathbf{H}| \mathrm{d} x \mathrm{~d} t .
$$

The general case easily follows from this inequality by means of an appropriate transformation of coordinates. 


\section{Whitney covering}

Lemma C.1 (covering lemma). Let $E$ be a non-empty, open, bounded subset of $\mathbb{R}^{n+1}$ equipped with the metric $d_{\alpha}$. Then there exists a family of balls with respect to the metric $d_{\alpha}\left\{Q_{r_{i}^{\alpha}}^{\alpha}\left(X_{i}^{\alpha}\right)\right\}_{i \in \mathbb{N}}$ such that

(a) $E=\bigcup_{i=1}^{\infty} Q_{\frac{r_{i}^{\alpha}}{2}}^{\alpha}\left(X_{i}^{\alpha}\right)=\bigcup_{i=1}^{\infty} Q_{r_{i}^{\alpha}}^{\alpha}\left(X_{i}^{\alpha}\right)$;

(b) $8 r_{i}^{\alpha} \leq d\left(X_{i}^{\alpha}, \partial E\right)<16 r_{i}^{\alpha} \quad \forall i \in \mathbb{N}$ with $0<r_{i}^{\alpha}<1$;

(c) $r_{j}^{\alpha}>2 r_{i}^{\alpha} \Longrightarrow Q_{r_{j}^{\alpha}}^{\alpha}\left(X_{j}^{\alpha}\right) \cap Q_{r_{i}^{\alpha}}^{\alpha}\left(X_{i}^{\alpha}\right)=\emptyset$;

(d) $Q_{\frac{r_{i}^{\alpha}}{4}}^{\alpha}\left(X_{i}^{\alpha}\right) \cap Q_{\frac{r_{j}^{\alpha}}{4}}^{\alpha}\left(X_{j}^{\alpha}\right)=\emptyset \quad \forall i, j \in \mathbb{N}, i \neq j$;

(e) $\#\left\{j \in \mathbb{N} \mid Q_{4 r_{j}^{\alpha}}^{\alpha}\left(X_{j}^{\alpha}\right) \cap Q_{4 r_{i}^{\alpha}}^{\alpha}\left(X_{i}^{\alpha}\right) \neq \emptyset\right\} \leq(120)^{n+2} \quad \forall i \in \mathbb{N}$.

Proof. For notational simplicity we skip the subscripts and superscripts $\alpha$. Since $E$ is bounded there exists a smallest number $k_{0} \in \mathbb{Z}$ such that the set $\{X \in$ $\left.E \mid d_{\alpha}(X, \partial E) \geq 2^{-k_{0}+4}\right\}$ is non-empty. For that number we define

$$
D_{k_{0}}:=\left\{X \in E \mid d_{\alpha}(X, \partial E) \geq 2^{-k_{0}+4}\right\} .
$$

Clearly, there exists a mostly countable set of points $\left\{X_{j}^{k_{0}}\right\}_{j \in \mathcal{J}_{k_{0}}} \subset D_{k_{0}}$ fulfilling

$$
d\left(X_{i}^{k_{0}}, X_{j}^{k_{0}}\right) \geq 2^{-k_{0}} \quad \forall i, j \in \mathcal{J}_{k_{0}},(i \neq j), \quad \bigcup_{j \in \mathcal{J}_{k_{0}}} Q_{2^{-k_{0}}}\left(X_{j}^{k_{0}}\right) \supset D_{k_{0}} .
$$

We define

$$
\tilde{D}_{k_{0}}:=\bigcup_{j \in \mathcal{J}_{0}} Q_{2^{-k_{0}}}\left(X_{j}^{k_{0}}\right) .
$$

From the construction it is immediately clear that

$$
D_{k_{0}} \subset \tilde{D}_{k_{0}} \subset E .
$$

Next, for $k \in \mathbb{Z}\left(k>k_{0}\right)$ we define the $D_{k},\left\{X_{j}^{k}\right\}_{\mathcal{J}_{k}}$ and $\tilde{D}_{k}$ recursively. Assume that $D_{\ell},\left\{X_{j}^{\ell}\right\}_{\mathcal{J}_{\ell}}$ and $\tilde{D}_{\ell}$ has already been defined for $\ell=1, \ldots, k-1$. Then we set

$$
D_{k}:=\left\{X \in E \backslash \bigcup_{\ell=1}^{k-1} \tilde{D}_{\ell} \mid d(X, \partial E) \geq 2^{-k+4}\right\} .
$$

There exists $\left\{X_{j}^{k}\right\}_{j \in \mathcal{J}_{k}} \subset D_{k}$ such that

$$
d\left(X_{i}^{k}, X_{j}^{k}\right) \geq 2^{-k} \quad \forall i, j \in \mathcal{J}_{k}(i \neq j), \quad \bigcup_{j \in \mathcal{J}_{k}} Q_{2^{-k}}\left(X_{j}^{k}\right) \supset D_{k} .
$$


Then we set

$$
\tilde{D}_{k}:=\bigcup_{j \in \mathcal{J}_{k}} Q_{2^{-k}}\left(X_{j}^{k}\right)
$$

Obviously,

$$
D_{k} \subset \tilde{D}_{k} \subset E
$$

Now, we define

$$
r_{\ell, j}:=2^{-\ell+1} \quad \ell \in \mathbb{N}, \quad j \in \mathcal{J}_{\ell}
$$

From the construction above it follows that

$$
\bigcup_{\ell=1}^{\infty} \bigcup_{j \in \mathcal{J}_{\ell}} Q_{\frac{r_{\ell, j}}{2}}\left(X_{j}^{\ell}\right)=E .
$$

This proves the first part of (a).

Moreover, it is readily seen that

$$
d\left(X_{j}^{\ell}, \partial E\right) \geq 2^{-\ell+4}=8 r_{\ell, j} \quad \forall \ell \in \mathbb{N}, \forall j \in \mathcal{J}_{\ell}
$$

Furthermore, as $X_{j}^{\ell} \notin \tilde{D}_{\ell-1}$ for $\ell \in \mathbb{N}\left(\ell>k_{0}\right)$ we deduce

$$
d\left(X_{j}^{\ell}, \partial E\right)<2^{-\ell+5}=16 r_{\ell, j} \quad \forall j \in \mathcal{J}_{\ell} .
$$

Hence, the property (b) follows from (C.2) and (C.3).

If $r_{j}^{\alpha}>2 r_{i}^{\alpha}$ then there exists $j \in \mathcal{J}_{\ell}$ and $i \in \mathcal{J}_{k}$ with $k \geq \ell+2$. We claim that

$$
Q_{r_{\ell, j}}\left(X_{j}^{\ell}\right) \cap Q_{r_{k, i}}\left(X_{i}^{k}\right)=\emptyset .
$$

Indeed, assuming there exists $\tilde{X} \in Q_{r_{\ell, j}}\left(X_{j}^{\ell}\right) \cap Q_{r_{k, i}}\left(X_{i}^{k}\right)$, since $X_{i}^{k} \notin D_{\ell+1}$, we have $d\left(X_{i}^{k}, \partial E\right) \leq 2^{-\ell+3}$. Thus,

$$
d(\tilde{X}, \partial E) \leq d\left(\tilde{X}, X_{i}^{k}\right)+d\left(X_{i}^{k}, \partial E\right) \leq 2^{-k+1}+2^{-\ell+3} \leq 2^{-\ell-1}+2^{-\ell+3} .
$$

On the other hand, we estimate

$$
d(\tilde{X}, \partial E) \geq d\left(X_{j}^{\ell}, \partial E\right)-d\left(\tilde{X}, X_{j}^{\ell}\right) \geq 2^{-\ell+4}-2^{-\ell+1} .
$$

However, the latter inequality contradicts to the former by means of

$$
2^{-\ell-1}+2^{-\ell+3}<2^{-\ell+4}-2^{-\ell+1} \text {. }
$$

Whence, (c).

To prove (d) let $\ell \in \mathbb{N}$ and $j \in \mathcal{J}_{\ell}$ be fixed. Assume for some $k \in \mathbb{N}$ and $i \in \mathcal{J}_{k}$ there exists $\tilde{X} \in E$ with

$$
\tilde{X} \in Q_{\frac{r_{\ell, j}}{4}}\left(X_{j}^{\ell}\right) \cap Q_{\frac{r_{k, i}}{4}}\left(X_{i}^{k}\right) .
$$


According to (c) this is only possible either for $k=\ell-1, k=\ell$ or $k=\ell+1$. Let us begin with the case $k=\ell$. By the triangular inequality

$$
d\left(X_{j}^{\ell}, X_{i}^{\ell}\right)<\frac{r_{\ell, j}}{4}+\frac{r_{\ell, i}}{4}=2^{-\ell}
$$

which can only be true if $i=j$ due to (C.1). In case $k=\ell+1$ again using triangular inequality one finds

$$
d\left(X_{j}^{\ell}, X_{i}^{\ell+1}\right)<\frac{r_{\ell, j}}{4}+\frac{r_{\ell+1, i}}{4}=\frac{3}{4} 2^{-\ell} .
$$

But since $X_{i}^{\ell+1} \notin \tilde{D}_{\ell}$ we must have $d\left(X_{j}^{\ell}, X_{i}^{\ell+1}\right) \geq 2^{-\ell}$, which is a contradiction. By an analogous reasoning one gets a contradiction in the case $k=\ell-1$. Consequently, $k=\ell$ and $i=j$. This completes the proof of (d).

In order to prove (e) let us denote for some fixed $i, k \in \mathbb{N}$

$$
B_{k, i}:=\left\{(j, \ell) \in \mathbb{N}^{2} \mid j \in \mathcal{J}_{\ell}, Q_{4 r_{\ell, j}}\left(X_{j}^{\ell}\right) \cap Q_{4 r_{k, i}}\left(X_{i}^{k}\right) \neq \emptyset\right\} .
$$

Due to property (d) we have for all $(j, \ell),(m, q) \in B_{k, i}$ with $(j, \ell) \neq(m, q)$ that $Q_{\frac{r_{\ell, j}}{4}}\left(X_{j}^{\ell}\right) \cap Q_{\frac{r_{m, q}}{4}}\left(X_{q}^{m}\right)=\emptyset$. For all $(j, \ell) \in B_{k, i}$ we estimate using (b)

$$
\begin{aligned}
8 r_{k, i} \leq d_{\alpha}\left(X_{i}^{k}, \partial E\right) & \leq d_{\alpha}\left(X_{i}^{k}, X_{j}^{\ell}\right)+d_{\alpha}\left(X_{j}^{\ell}, \partial E\right) \\
& \leq d_{\alpha}\left(X_{i}^{k}, X\right)+d_{\alpha}\left(X_{j}^{\ell}, X\right)+16 r_{\ell, j} \leq 4 r_{k, i}+20 r_{\ell, j}
\end{aligned}
$$

Hence, $r_{\ell, j} \leq 5 r_{k, i}$. From this follows that $Q_{\frac{r_{\ell, j}}{4}}\left(X_{j}^{\ell}\right) \subset Q_{30 r_{k, i}}\left(X_{i}^{k}\right)$. These two properties and $r_{\ell, j} \geq \frac{1}{2} r_{k, i}$ imply

$$
\begin{aligned}
\mathcal{L}_{n+1}\left(Q_{30 r_{k, i}}\left(X_{i}^{k}\right)\right) & \geq \sum_{(j, \ell) \in B_{k, i}} \mathcal{L}_{n+1}\left(Q_{\frac{r_{\ell, j}}{4}}\left(X_{j}^{\ell}\right)\right) \\
& \geq \frac{1}{120^{n+2}} \#\left(B_{k, i}\right) \mathcal{L}_{n+1}\left(Q_{30 r_{k, i}}\left(X_{i}^{k}\right)\right)
\end{aligned}
$$

where we also used the properties of the Lebesgue measure and the metric $d_{\alpha}$. This immediately yields (e).

An appropriate relabeling of the points $X_{i}^{k}$ and the radii $r_{k, i}$ proves the lemma.

\section{References}

[1] E. ACERBI and N. FusCO, Semicontinuity problems in the calculus of variations, Arch. Ration. Mech. Anal. 86 (1984), 125-145. 
[2] H. Amann, Stability of the rest state of a viscous incompressible fluid, Arch. Ration. Mech. Anal. 126 (1994), 231-242.

[3] G. Astarita and G. Marucci, "Principles of non-Newtonian Fluid Mechanics", McGraw-Hill, London, 1974.

[4] G. K. Batchelor, "An Introduction to Fluid Dynamics", Cambridge University Press, Cambridge, 1967.

[5] H. Bellout, F. Bloom and J. NeČAS, Young measure-valued solutions for non-Newtonian incompressible fluids, Comm. Partial Differential Equations 19 (1994), 1763-1803.

[6] R. B. Bird, R. C. ARMStrong and O. Hass Ager, "Dynamic of Polymer Liquids", John Wiley, 1987, 2nd edition.

[7] D. Bothe and J. PRÜSs, $L^{p}$-theory for a class of non-Newtonian fluids, SIAM J. Math. Anal. 39 (2007), 379-421.

[8] D. Cioranescu, Quelques exemples de fluides newtoniens generalisés, In: "Mathematical Topics in Fluid Mechanics" (Lisbon, 1991) (Harlow), Pitman Res. Notes Math. Ser., Vol. 274, Longman Sci. Tech., Harlow, 1992, 1-31.

[9] R. R. COIFMAN and G. WEISS, Extensions of Hardy spaces and their use in analysis, Bull. Amer. Math. Soc. 83 (1977), 569-645.

[10] H. BEIRÃO DA VEIGA, On the regularity of flows with Ladyzhenskaya shear-dependent viscosity and slip or nonslip boundary conditions, Comm. Pure Appl. Math. 58 (2005), 552-577.

[11] H. BeIRÃO DA VEIGA, P. KAPLICKÝ and M. RŮŽIČKA, Boundary regularity of shearthickening flows, J. Math. Fluid Mech. (2010), to appear.

[12] M. DE GUZMÁN, "Differentiation of Integrals in $R^{n}$ ", Springer-Verlag, Berlin, 1975, with appendices by Antonio Córdoba, and Robert Fefferman, and two by Roberto Moriyón, Lecture Notes in Mathematics, Vol. 481.

[13] L. Diening, J. MÁLeK and M. Steinhauer, On Lipschitz truncations of Sobolev functions (with variable exponent) and their selected applications, ESAIM Control Optim. Calc. Var. 14 (2008), 211-232, DOI: 10.1051/cocv:2007049.

[14] L. DIENING and M. RŮŽIČKA, Strong solutions for generalized Newtonian fluids, J. Math. Fluid Mech. 7 (2005), 413-450.

[15] J. Frehse, J. MáleK and M. Steinhauer, An existence result for fluids with shear dependent viscosity - steady flows, Nonlinear Anal. 30 (1997), 3041-3049.

[16] J. Frehse, J. MÁLEK and M. SteinhAUER, On existence result for fluids with shear dependent viscosity - unsteady flows, In: "Partial Differential Equations", W. Jäger, J. Nečas, O. John, K. Najzar and J. Stará (eds.), Chapman and Hall, 2000, 121-129.

[17] J. Frehse, J. Málek and M. SteinhAUer, On analysis of steady flows of fluids with shear-dependent viscosity based on the Lipschitz truncation method, SIAM J. Math. Anal. 34 (2003), 1064-1083 (electronic).

[18] M. FUCHS, On stationary incompressible Norton fluids and some extensions of Korn's inequality, Z. Anal. Anwendungen 13 (1994), 191-197.

[19] G. P. GALDI, "An Introduction to the Mathematical Theory of the Navier-Stokes Equations, Linearized Steady Problems", Tracts in Natural Philosophy, Vol. 38, Springer, New York, 1994.

[20] G. P. Galdi, C. G. Simader and H. Sohr, On the Stokes problem in Lipschitz domains, Ann. Mat. Pura Appl. (4) 167 (1994), 147-163.

[21] D. Gilbarg and N. S. Trudinger, "Elliptic Partial Differential Equations of Second Order", Springer, Berlin, 2001, Reprint of the 1998 edition.

[22] E. HopF, Über die Anfangswertaufgabe für die hydrodynamischen Grundgleichugen, Math. Nachr. 4 (1951), 213-231.

[23] R. R. Huilgol, "Continuum Mechanics of Viscoelastic Liquids", Hindustan Publishing Corporation, Delhi, 1975.

[24] J. KinNUNEN and J. L. LEWIS, Very weak solutions of parabolic systems of p-Laplacian type, Ark. Mat. 40 (2002), 105-132. 
[25] O. A. LADYZHEnskaya, New equations for the description of motion of viscous incompressible fluids and solvability in the large of boundary value problems for them, Proc. Steklov Inst. Math. 102 (1967), 95-118.

[26] O. A. LADYZHENSKAYA, Modifications of the Navier-Stokes equations for large gradients of the velocities, Zap. Naučn. Sem. Leningrad. Otdel. Mat. Inst. Steklov. (LOMI) 7 (1968), $126-154$.

[27] O. A. LADYZHENSKAYA, On some modifications of the Navier-Stokes equations for large gradients of velocity, Zap. Naučn. Sem. Leningrad. Otdel. Mat. Inst. Steklov (LOMI) 7 (1968), 126-154.

[28] O. A. LADYZhensKaya, "The Mathematical Theory of Viscous Incompressible Flow", Gordon and Breach, New York, 1969, 2nd edition.

[29] J. LERAY, Sur le mouvement d'un liquide visqueux emplissant l'espace, Acta Math. 63 (1934), 193-248.

[30] J. L. Lions, "Quelques Méthodes de Résolution des Problèmes aux Limites Non Linéaires", Dunod, Paris, 1969.

[31] J. MÁLEK, J. NeČAS and A. NovotnÝ, Measure-valued solutions and asymptotic behavior of a multipolar model of a boundary layer, Czechoslovak Math. J. 42 (117) (1992), 549-576.

[32] J. MÁlek, J. NeČAs, M. Rokyta and M. RŮŽIČKA, "Weak and Measure-valued Solutions to Evolutionary PDEs", Applied Mathematics and Mathematical Computations, Vol. 13, Chapman \& Hall, London, 1996.

[33] J. MÁleK, J. NeČAS and M. RŮŽIČKA, On the non-Newtonian incompressible fluids, Math. Models Methods Appl. Sci. 3 (1993), 35-63.

[34] J. MÁleK, J. NEČAS and M. RŮŽIČKA, On Weak Solutions to a Class of Non-Newtonian Incompressible Fluids in Bounded Three-dimensional Domains. The Case $p \geq 2$, Adv. Differential Equations 6 (2001), 257-302.

[35] J. MÁLEK and K. R. RAJAGOPAL, Mathematical issues concerning the Navier-Stokes equations and some of its generalizations, Handb. Differential Equations, Elsevier/NorthHolland, Amsterdam, 2005, 371-459.

[36] J. MÁLEK, K. R. RAJAGOPAL and M. RŮŽIČKA, Existence and regularity of solutions and the stability of the rest state for fluids with shear dependent viscosity, Math. Models Methods Appl. Sci. 5 (1995), 789-812.

[37] J. MALÝ and W. P. ZIEMER, "Fine Regularity of Solutions of Elliptic Partial Differential Equations", Mathematical Surveys and Monographs, Vol. 51, American Mathematical Society, Providence, RI, 1997.

[38] J. NAUMANN and M. WOLFF, Interior integral estimates on weak solutions of nonlinear parabolic systems, 1994, preprint Humboldt University.

[39] J. NEČAS, Sur le normes équivalentes dans $W_{p}^{k}(\Omega)$ et sur la coercivité des formes formellement positives, Séminaire Equations aux Dérivées Partielles, Montreal 317 (1966), 102128.

[40] J. NeČAS, "Les Méthodes Directes en la Thèorie des Equations Elliptiques", Academia, Praha, 1967.

[41] J. NEČAS, Theory of multipolar viscous fluids, In: "The Mathematics of Finite Elements and Applications", VII (Uxbridge, 1990) (London), Academic Press, London, 1991, 233-244.

[42] C. PARÉS, Existence, uniqueness and regularity of solution of the equations of a turbulence model for incompressible fluids, Appl. Anal. 43 (1992), 245-296.

[43] G. Da Prato, Spazi $\mathcal{L}^{(p, \theta)}(\Omega, \delta)$ e loro proprietà, Ann. Mat. Pura Appl. (4) 69 (1965), 383-392.

[44] K. R. Rajagopal, Mechanics of Non-Newtonian Fluids, In: "Recent Developments in Theoretical Fluid Mechanics", G. P. Galdi and J. Nečas (eds.), Research Notes in Mathematics Series, Vol. 291, Longman, 1993, 129-162. 
[45] M. RŮŽIČKA, Flow of shear dependent electrorheological fluids: unsteady space periodic case, In: "Applied Nonlinear Analysis", A. Sequeira (ed.), Kluwer/Plenum, New York, 1999, 485-504.

[46] M. RŮŽIČKA, Modeling, mathematical and numerical analysis of electrorheological fluids, Appl. Math. 49 (2004), 565-609.

[47] P. ShVARTSman, On extensions of Sobolev functions defined on regular subsets of metric measure spaces, J. Approx. Theory 144 (2007), 139-161.

[48] E. M. SteIn, "Singular Integrals and Differentiability Properties of Functions", Princeton University Press, Princeton, N.J., 1970.

[49] M. STRUWE, On the Hölder continuity of bounded weak solutions of quasilinear parabolic systems, Manuscripta Math. 35 (1981), 125-145.

[50] C. TRuesdell and W. Noll, "The Non-Linear Field Theories of Mechanics", Handbuch der Physik, Vol. III/3, Springer, New York, 1965.

[51] J. WOLF, "Regularität schwacher Lösungen elliptischer und parabolischer Systeme partieller Differentialgleichungen mit Entartung. Der Fall $1<p<2$ ", Dissertation, Humboldt Universität, 2001.

[52] J. WOLF, Existence of weak solutions to the equations of nonstationary motion of nonNewtonian fluids with shear-dependent viscosity, J. Math. Fluid Mech. 9 (2007), 104-138.

\author{
Universität Freiburg \\ Mathematisches Institut \\ Eckerstr, 1 \\ 79104 Freiburg, Germany \\ diening@mathematik.uni-freiburg.de \\ Universität Freiburg \\ Mathematisches Institut \\ Eckerstr, 1 \\ 79104 Freiburg, Germany \\ rose@mathematik.uni-freiburg.de \\ Otto-von-Guericke-Universität Magdeburg \\ Universitätsplatz 2 \\ Postfach 4120 \\ 39106 Magdeburg, Germany \\ joerg.wolf@ovgu.de
}

